INTER NATIONAL MONETARY FUND
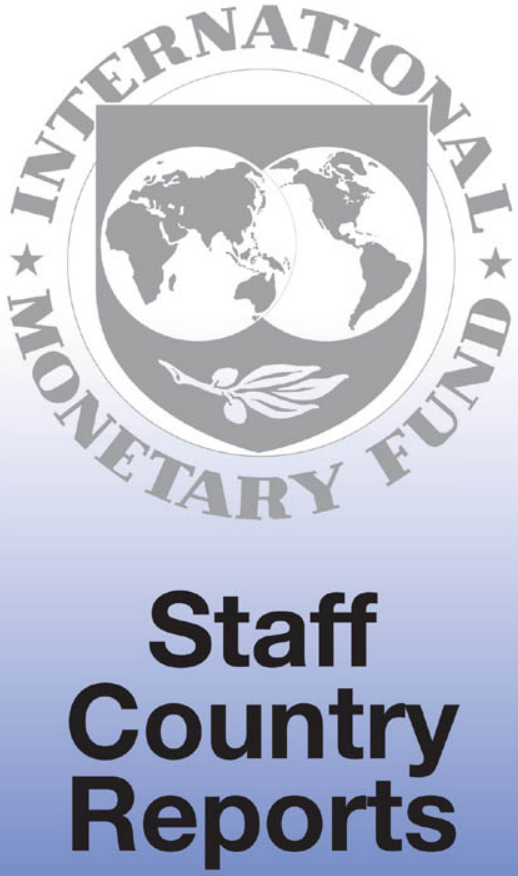


\section{Georgia: Ninth Review Under the Stand-By Arrangement and Request for Waiver of Nonobservance of Performance Criterion-Staff Report; Staff Supplement; Press Release on the Executive Board Discussion; and Statement by the Executive Director for Georgia.}

In the context of the Ninth Review Under the Stand-By Arrangement and Request for Waiver of Nonobservance of Performance Criterion, the following documents have been released and are included in this package:

- $\quad$ The staff report for the Ninth Review Under the Stand-By Arrangement and Request for Waiver of Nonobservance of Performance Criterion, prepared by a staff team of the IMF, following discussions that ended on April 28, 2011, with the officials of Georgia on economic developments and policies. Based on information available at the time of these discussions, the staff report was completed on May 24, 2011. The views expressed in the staff report are those of the staff team and do not necessarily reflect the views of the Executive Board of the IMF.

- $\quad$ A Press Release summarizing the views of the Executive Board as expressed during its June 8, 2011 discussion of the staff report that completed the request and/or review.

- $\quad$ A statement by the Executive Director for Georgia.

The document listed below has been or will be separately released.

Letter of Intent sent to the IMF by the authorities of Georgia*

*Also included in Staff Report

The policy of publication of staff reports and other documents allows for the deletion of market-sensitive information.

Copies of this report are available to the public from

International Monetary Fund $\bullet$ Publication Services $70019^{\text {th }}$ Street, N.W. $\bullet$ Washington, D.C. 20431

Telephone: (202) 623-7430 • Telefax: (202) 623-7201

E-mail: publications@imf.org Internet: http://www.imf.org

\section{International Monetary Fund Washington, D.C.}


INTERNATIONAL MONETARY FUND

\title{
GEORGIA
}

\section{Ninth Review Under the Stand-By Arrangement and Request for Waiver of Nonobservance of Performance Criterion}

\author{
Prepared by the Middle East and Central Asia Department \\ (In consultation with other departments)
}

Approved by David Owen (MCD) and Dominique Desruelle (SPR)

May 24, 2011

- Background: The present review is the final review under the Stand-By Arrangement (SBA). The seventh and eighth reviews were concluded on January 12, 2011. Access under the SBA is SDR 747.1 million (497.1 percent of quota), of which SDR 577.1 million has been disbursed. Since the last drawing in July 2010, the authorities have treated the arrangement as precautionary. The arrangement expires June 14, 2011.

- Strategy for the remainder of 2011: The stabilization objectives supported by the current SBA have been largely achieved. A successful Eurobond issue has eased considerably the debt service hump of 2013. The authorities' policy targets for the remainder of 2011 are in line with those presented at the seventh and eight reviews. The authorities are elaborating a medium-term policy framework, in support of which they intend to request a successor Fund arrangement.

- Team: E. Gardner (head and Senior Resident Representative), E. Martin, A. Luca (all MCD), M. Gerard (FAD), I. Halikias (SPR), and N. Sharashidze (Resident Representative Office).

- Exchange Rate Regime: The regime is classified as "floating."

- Statistics: Economic data are broadly adequate for surveillance and program monitoring. Georgia participates in the GDDS and subscribed to the SDDS in May 2010. 


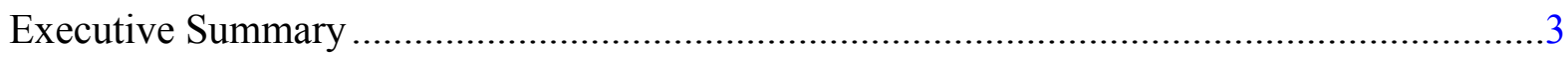

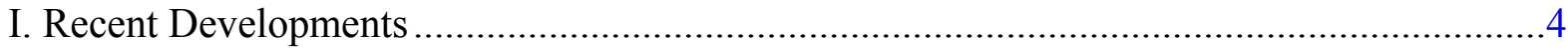

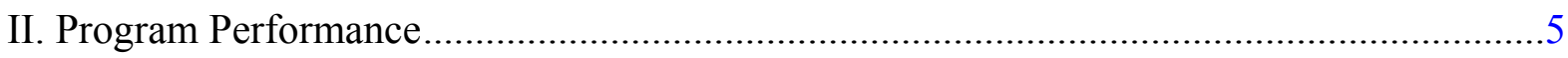

III. Macroeconomic Outlook and Policies for the Remainder of 2011 .....................................6

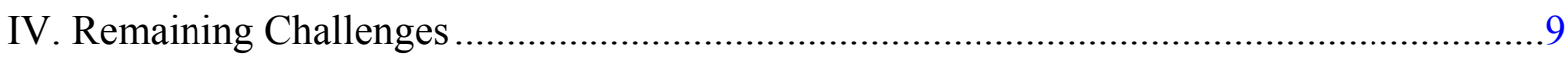

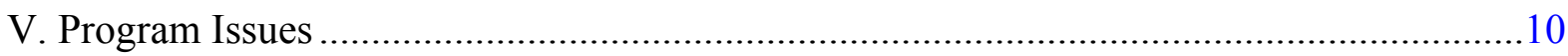

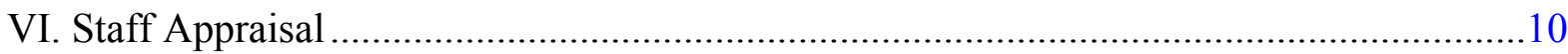

\section{TABLES}

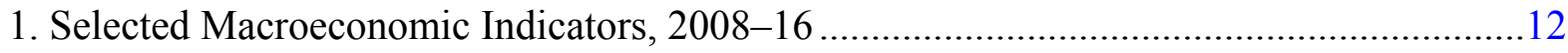

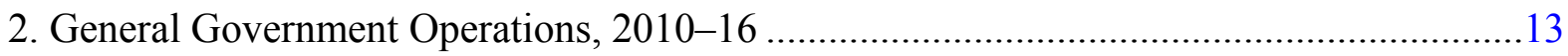

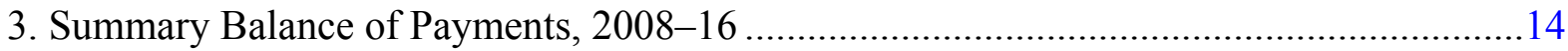

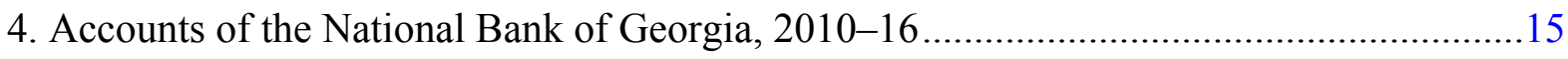

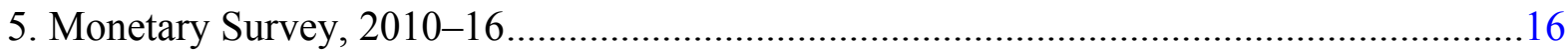

6. Selected Monetary and Financial Soundness Indicators, 2008-11 …..............................17

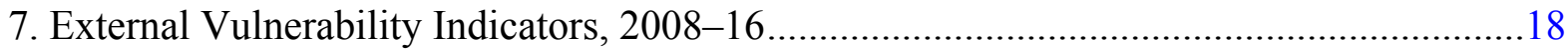

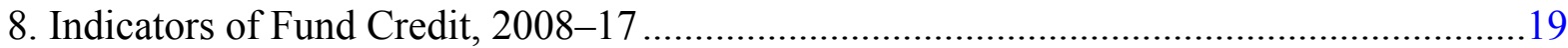

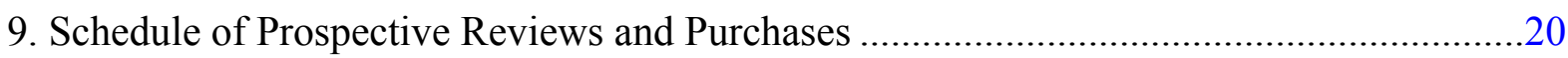

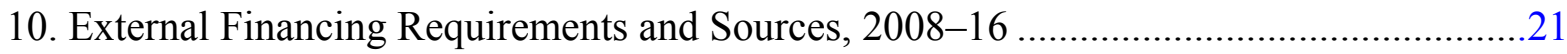

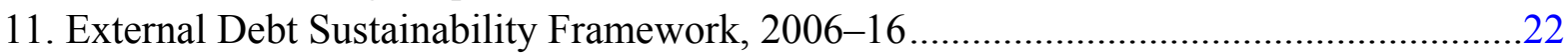

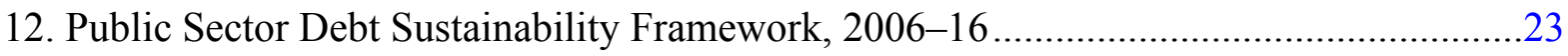

\section{FIGURES}

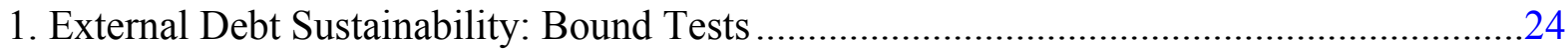

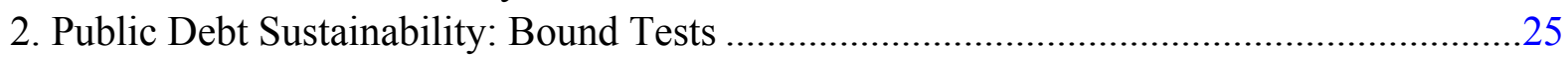

\section{AtTaChment}

I. Letter of Intent. 


\section{EXECUTIVE SUMMARY}

\section{Recent economic developments have been largely in line with program expectations.} Economic activity indicators support the real GDP growth projection of 51/2 percent in 2011. The inflation projection has been revised up (to $8 \frac{1}{2}$ percent by end-year) on the back of rising food and fuel prices, but core inflation remains subdued. Balance of payment developments have been more favorable than anticipated in the first quarter owing to higher private inflows, including tourism. The current account deficit is still projected to widen moderately in 2011 (to 10.8 percent of GDP) on account of higher import prices and to decline steadily thereafter. In April, the government issued a \$500-million Eurobond at very favorable terms. The proceeds were used to redeem, through a voluntary exchange, $\$ 435$ million of the $\$ 500$-million Eurobond maturing in 2013, reducing significantly the external debt rollover hump of 2013.

Policy targets for 2011 have been strengthened. Reflecting the stronger balance of payments developments of the first quarter, NIR is now expected to increase by an additional $\$ 205$ million (to $\$ 1.1$ billion) relative to the previous review. The 2011 fiscal deficit is now projected at 3.6 percent of GDP (rather than 4.3 percent), while allowing for an increase in social spending to alleviate the social impact of higher fuel and food prices. Faster deficit reduction largely reflects one-off revenues, and the underlying structural adjustment remains as was envisaged at the time of the seventh and eighth reviews. The monetary policy stance remains consistent with a steady deceleration of inflation in the second half of the year.

\section{All end-March performance criteria (PCs) were met with the exception of the ceiling on} government expenditure. The expenditure overrun stems from faster execution of capital spending and increased social spending to mitigate the impact of rising food and energy prices. Staff supports the request for a waiver, given that most of the higher-than-expected revenues were saved (as reflected in the lower deficit target for 2011), and the underlying path of fiscal consolidation remains on track.

\section{Challenges remain notwithstanding the significant progress made under the SBA in} stabilizing the economy and restoring confidence. The large current account deficit remains a source of vulnerability, and additional fiscal adjustment is required to stabilize public debt, all the while meeting important social spending priorities. The authorities are in the process of designing a medium-term policy framework to address these challenges. 


\section{RECENT DEVELOPMENTS}

\section{Economic and financial developments are largely in line with the outlook} discussed in the staff report for the 2011 Article IV consultation (Country Report No. 11/87):

- Strong first quarter economic activity indicators, including buoyant tax receipts, are consistent with the real GDP growth projection of $5 \frac{1}{2}$ percent in 2011.

- Year-on-year inflation peaked at 13.9 percent in March before edging down to 13.5 percent in April. Core inflation remained subdued, at 1.5 percent in April. High food and energy inflation reflects recent commodity price hikes and is expected to decline through the rest of the year. ${ }^{1}$

- Balance of payment developments have been more favorable than anticipated. While higher import prices contributed to a sharp widening of the trade deficit in the first quarter, other inflows turned out stronger than projected, in particular tourism (40 percent growth year-on-year

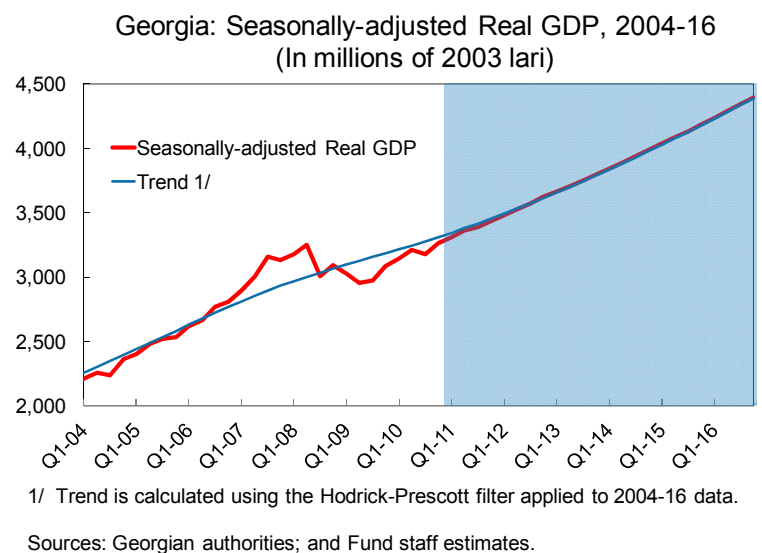

Georgia: Measures of Consumption Prices, 2005-11 (In percent, year-on-year inflation)

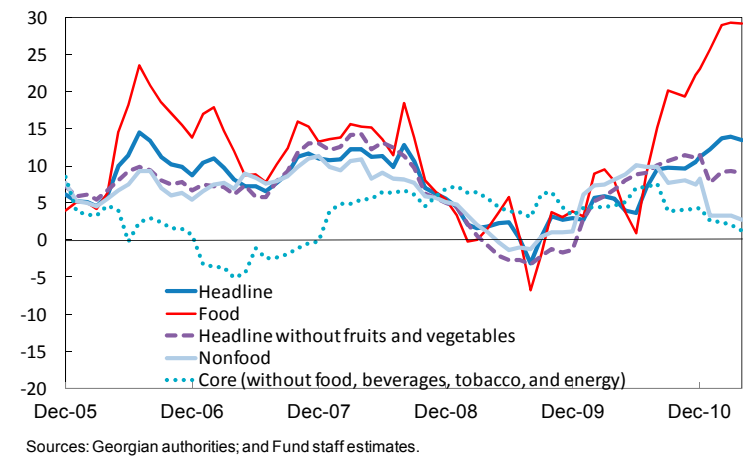
in the first quarter) and net private capital inflows.

- Favorable external developments contributed to an appreciation of the lari against the dollar, while allowing the central bank to make net purchases on the foreign exchange market. During the first four months of the year, the lari appreciated by 7.6 percent against the dollar and by 3.3 percent in nominal effective terms. Depreciation pressures reemerged in May, in connection to large-scale private foreign exchange purchases by one bank (to finance a Eurobond buyback) and by non-financial corporates. The central bank sold dollars at auction to avoid excessive volatility, but also allowed the rate to depreciate. Since the beginning of the year, the central bank has purchased \$20 million, on a net basis, on the foreign exchange market, and net international reserves have now reached their pre-crisis level.

\footnotetext{
${ }^{1}$ Food and energy prices make up 39 and 13 percent, respectively, of the total CPI basket.
} 
- On April 7, the government issued a \$500-million Eurobond at very favorable terms (10-year maturity and 7.125-percent yield). The issue was used to redeem, through a voluntary exchange, $\$ 435$ million of the $\$ 500$-million Eurobond maturing in 2013 , reducing significantly the external debt rollover hump of 2013.

- Money growth has decelerated as expected after the post-crisis surge, while credit continues to grow at a healthy rate. Notwithstanding the improvement in banks' liquidity conditions, lari deposit and lending rates have remained stable, owing in part to steady monetary tightening since June 2010 . The dollar lending rate has been declining slowly, after rising earlier this year in response to a tightening of foreign exchange reserve requirements. The banks' financial situation has strengthened further: NPLs have declined to 5 percent (IMF definition) in March, reflecting writeoffs and an improved environment.
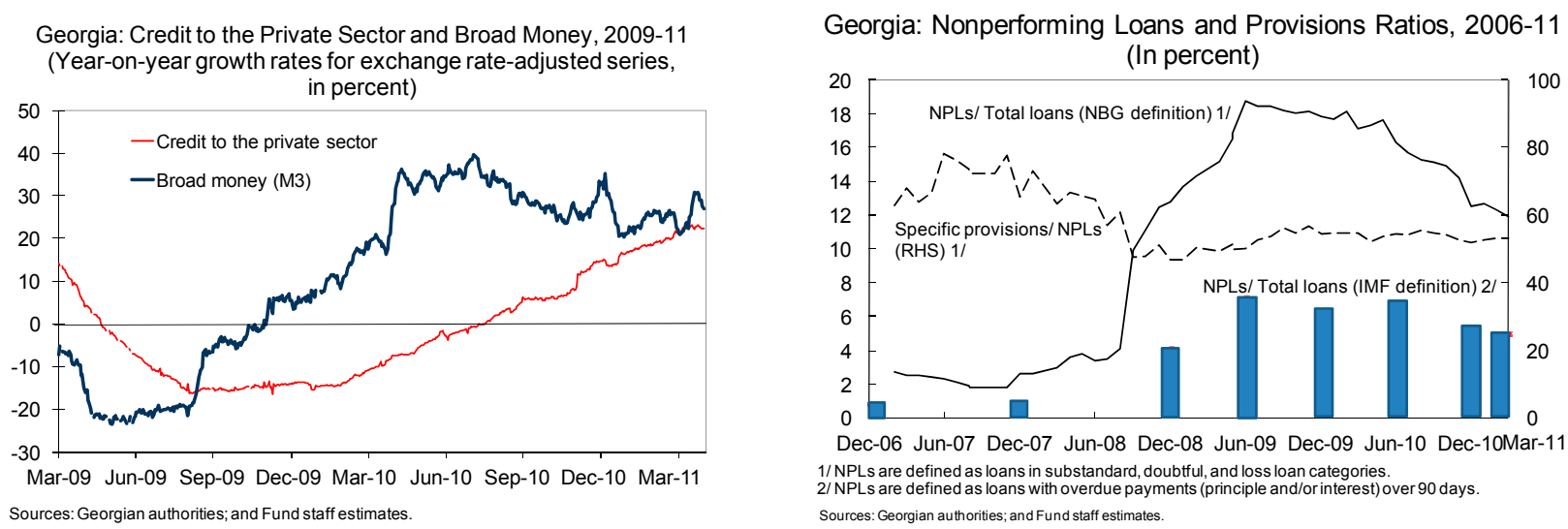

- In April, the government started issuing 5-year T-notes, a significant step in their strategy to lengthen government paper maturities.

\section{Program Performance}

\section{End-March 2011 PCs were met, with the exception of the general government expenditure ceiling:}

- The end-March PCs on NIR and NDA were observed by wide margins, owing to lower-than-programmed foreign exchange intervention, a large one-off tax payment in U.S. dollars by an exporter, and lower reserve money growth in the case of NDA.

- The end-March PC on the government deficit was observed by a wide margin (1.4 percent of annual GDP) as higher-than-projected revenues were only partly offset by higher spending. 
- The end-March PC on government expenditure was missed by about 0.2 percent of annual GDP, owing to an accelerated implementation of investment projects and an increase in social spending (electricity and food vouchers) aimed at alleviating the impact of the hike in food prices. ${ }^{2}$ Other current spending was lower than expected, but not enough to offset these two factors. A waiver of nonobservance is requested (LOI $\uparrow 10$ ) because of corrective actions by the authorities, in the form of their commitment to reduce the overall 2011 fiscal deficit by more than envisaged at the time of the seventh and eighth reviews, and thereby to deliver the structural adjustment envisaged under the program. ${ }^{3}$

- The end-March indicative target on the contracting and guaranteeing of external debt was met.

3. The end-December $\mathbf{2 0 1 0}$ fiscal deficit and government expenditure, for which data were not available at the time of the seventh and eighth reviews, were below projected levels. The corresponding PCs were missed by less than the amounts on the basis of which the waivers were granted.

\section{MaCroeconomic OUtlook ANd POLICIES FOR THE Remainder OF 2011}

4. Against an unchanged real GDP growth outlook, the inflation projection has been revised upward (to $81 \frac{1}{2}$ by end-year) on the back of higher food and fuel prices. In the absence of second-round effects, headline inflation is now expected to decelerate steadily in the second half of the year, as food and energy prices stabilize.

5. Because of the adverse terms-of-trade shock, the current account deficit is projected to widen in 2011 (by about 11/4 percentage points of GDP), but by much less than envisaged at the time of the Article IV consultation (about $2 \frac{1}{2}$ percentage points of GDP). This deterioration is expected to be temporary and masks an underlying improvement in the current account balance, measured at constant prices. With the expected stabilization of commodity prices, the current account deficit is projected to narrow steadily, driven by the improvement in the relative cyclical position of Georgia's trading partners, productivity gains, and the convergence of the real exchange rate toward its equilibrium level.

\footnotetext{
${ }^{2}$ Implementation of projects funded by the Millennium Challenge Georgia Fund was accelerated ahead of the expected end of the Millennium Challenge Compact in mid-2011.

${ }^{3}$ The ceiling on government expenditure was intended to ensure that cyclical and one-off revenue windfalls would not be spent in ways that would weaken the underlying structural fiscal adjustment.
} 

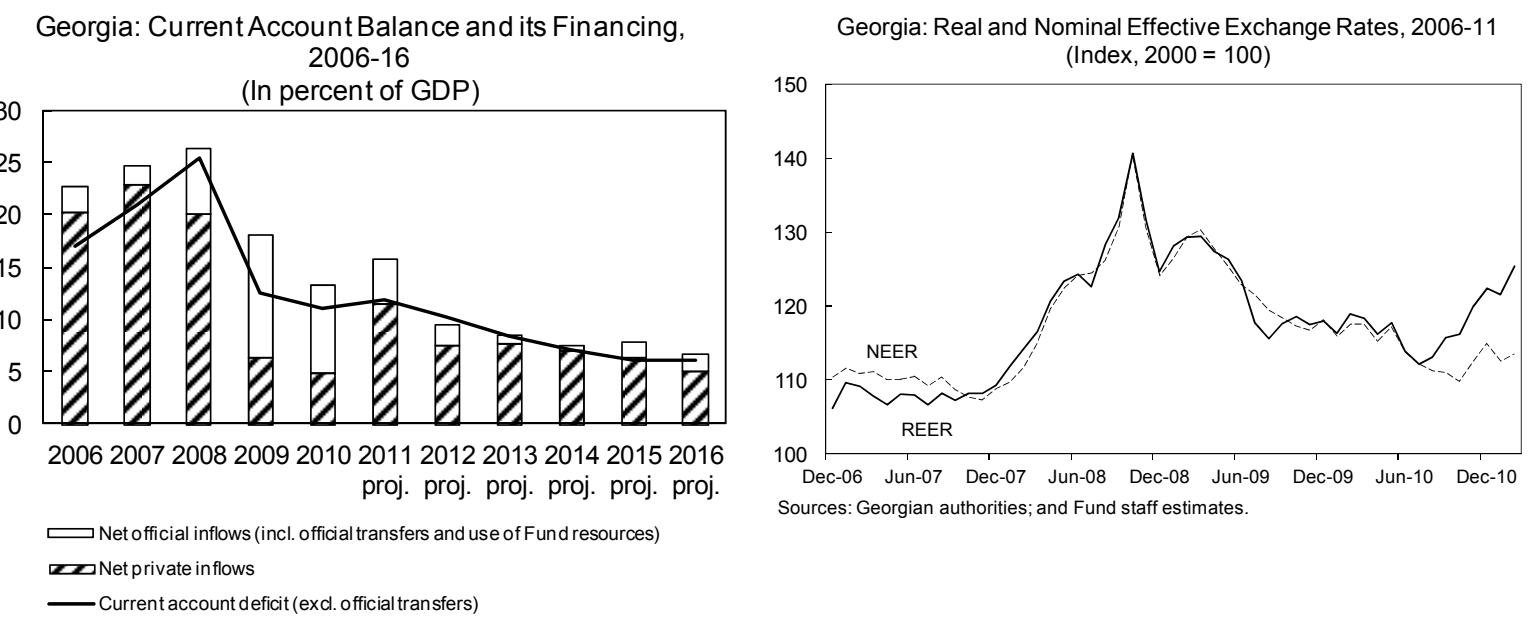

Sources: National Bank of Georgia; and Fund staff estimates.

\section{Consistent with the fiscal structural adjustment envisaged at the time of the last} review, the authorities intend to reduce the government's deficit to 3.6 percent of GDP in 2011, compared with 4.3 percent of GDP previously. This downward revision reflects an improvement in projected revenues (equivalent to 1.7 percentage point of GDP, of which 1.3 percentage point in tax revenues) owing to one-off factors (settlement of past tax arrears amounting to about 1 percentage point of GDP) and higher tax buoyancy already observed in the first quarter, and related in part to improved compliance owing to simplified tax procedures (e.g., web portal and online payment). So as to preserve the envisaged structural adjustment path, the one-off revenue would be used essentially to reduce the deficit and to finance additional one-off social outlays (food and electricity vouchers amounting to 0.2 percent of GDP) aimed at alleviating the impact of higher food and fuel prices. The structural component of the revenue increase and the residual one-off revenue would finance: a 25 percent increase in the basic pension to the equivalent of $\$ 60$ per month $(0.2$ percentage point of GDP), which will become effective in September; an increase in capital spending (0.6 percentage point of GDP); and an inflation-related increase in spending on goods and services. The authorities consider that an even better revenue performance is possible relative to the program scenario, and have adopted a supplementary budget that allows for higher capital spending (by 0.2 percent of GDP) if these additional revenues materialize.

Georgia: Overall and Structural Fiscal Balances, 2009-11

(In percent of GDP)

\begin{tabular}{|l|c|cc|cc|}
\hline & \multirow{2}{*}{2009} & \multicolumn{2}{|c|}{2010} & \multicolumn{2}{|c|}{2011} \\
\cline { 3 - 6 } & & $7 / 8$ th Rev. & Proj. & 7/8th Rev. & Proj. \\
\hline \multirow{2}{*}{ Overall balance } & -9.2 & -6.8 & -6.6 & -4.3 & -3.6 \\
Structural balance & -8.5 & -7.0 & -6.8 & -4.7 & -4.7 \\
\hline
\end{tabular}

Sources: Ministry of Finance; and Fund staff estimates. 
7. While the current fiscal projections do not factor in any revenue measures, the authorities retain the flexibility to introduce such measures should the need arise, notably if the expenditure containment objectives underpinning their fiscal consolidation strategy prove difficult to achieve. The constitutional amendment adopted in December 2010 subjecting tax increases to referendum requirement will become effective only after the 2013 presidential election. Also, the authorities reiterated their commitment to adopt an escape clause in line with staff recommendations (see Box 4 of Country Report No. 11/87) before this requirement becomes effective. This clause could be included, along with fiscal rules (ceilings) on budget spending, the fiscal deficit, and the public debt, in an organic budget law that the authorities are considering submitting to parliament later this year.

8. The authorities indicated that a further substantial increase in pensions might be necessary, but recognized the need to structure and finance any such increase in a way that does not compromise fiscal adjustment objectives. The current old-age pension system is not financed by contributions but by the state budget and provides a pension to all persons of pension age ( 65 for men and 60 for women) who are no longer working. It is part of a broader program that includes pensions for the disabled and war veterans, as well as targeted social assistance to the poor. Total benefits, including social assistance for nonpensioners, come to 4.6 percent of GDP, of which 4 percent of GDP is accounted by pensions. The basic pension is GEL80 per month (about \$50) and will be raised to GEL100 in September, but the authorities are considering another substantial increase in 2012. The mission emphasized the importance of containing the net budgetary cost of such an increase during the fiscal consolidation period. Based on the recommendations of a Fund technical assistance mission on pension reform that was present in Tbilisi at the same time, it laid out a number of options, including limiting (or phasing in) any increase through means testing. The authorities also enquired about the merits of introducing a contributory pension scheme. The mission stressed that, while such a scheme could be envisaged as a supplemental plan to the existing state pension system, any move in that direction is both difficult and fraught with risks, and should therefore be carefully assessed beforehand, and not tied to short-term considerations such as the need to finance (through the introduction of social contributions) increases in the basic state pension.

9. Given that headline inflation has started to decline earlier than projected and that core inflation remains subdued, the authorities consider the current monetary stance to be appropriate and consistent with a further deceleration of inflation in the second half of the year. While headline inflation is still high, reflecting elevated food and fuel prices, there have been so far no signs of second-round effects. Also, CD and government securities rates have declined across the yield curve since the beginning of the year, which the central bank attributes to declining inflation expectations. Nonetheless, the authorities restated their readiness to tighten the monetary stance in response to any signs of more persistent inflation and/or excessive growth in monetary and credit aggregates. The authorities and staff agreed that, should more tightening be needed, it should rely on a mix of 
policy rate adjustments and increases in reserve requirements on foreign currency liabilities, so as to avoid fueling further dollarization. ${ }^{4}$

\section{The authorities consider that a flexible exchange rate remains key to meeting} external adjustment objectives, while increasing NIR. The NBG's transactions on the foreign currency market this year have been guided by two considerations: opportunistic build-up of international reserves in the face of steady appreciation pressures; and the need to smooth out large-scale private transactions deemed as market disruptive. Based on an improved balance of payments outlook relative to the last review, the authorities have raised their NIR projection for 2011 by $\$ 205$ million, to $\$ 1.1$ billion. Looking ahead, the authorities and staff agreed that the foreign exchange policy should be guided by the objective of increasing NIR. Although a

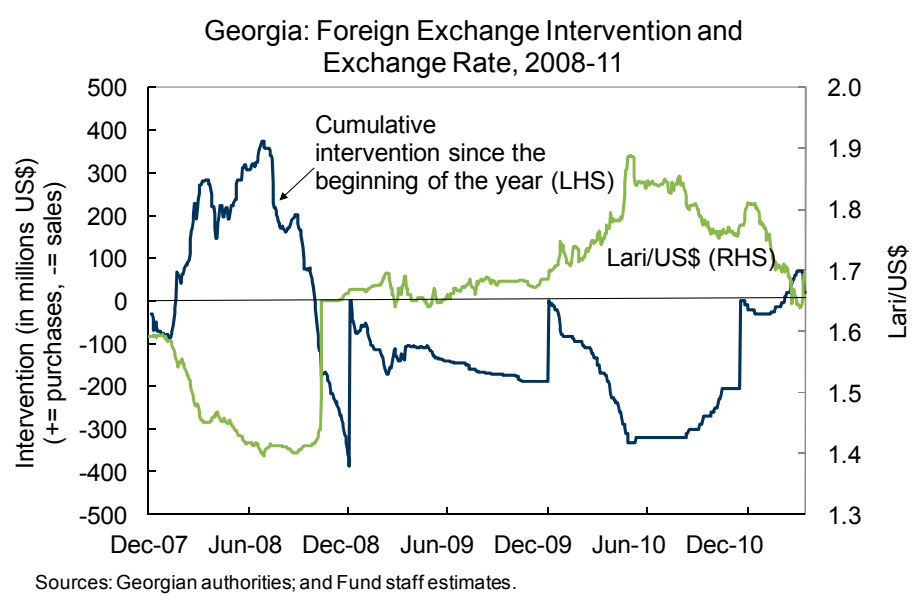
preannounced schedule of foreign currency purchases could achieve that objective while allowing the exchange rate to move freely, the authorities and the mission agreed that a move in this direction would be premature in view of the current shallowness of the market and thus potentially large volatility of the exchange rate.

\section{Remaining Challenges}

\section{Notwithstanding the significant progress made under the SBA in stabilizing the economy and restoring confidence, the authorities and staff agreed that a number of challenges remain:}

- The large current account deficit remains a source of vulnerability, especially when combined with rising external debt servicing obligations in 2013.

- A significant adjustment is still required to bring the primary fiscal deficit below its debt stabilizing level.

- Unemployment remains high and recent increases in the prices of basic foodstuff and oil have eroded real incomes, notably for the most vulnerable.

\footnotetext{
${ }^{4}$ The recent widening spread between lari and foreign currency deposit rates and appreciation of the lari against the U.S. dollar do not appear to have triggered a reallocation of savings toward the local currency.
} 
- Banks have overcome the stress created by the financial crisis, but potential new risks need to be monitored closely.

12. The authorities indicated their intention to tackle these challenges forcefully. They are in the process of designing a medium-term policy framework, whose broad parameters were discussed with staff and are reflected in the attached medium-term projections. This framework will notably aim at: addressing remaining macroeconomic vulnerabilities, and in particular, ensuring the return to a viable external position; ensuring that social safety nets address the impact of the rise in food and fuel prices on the most vulnerable; strengthening incentives for private sector investment in the tradable sector through structural reforms and supportive public spending; and containing financial sector risks as access to capital and credit improves.

\section{Program Issues}

13. The authorities reiterated their interest in a successor arrangement, but needed time to design their medium-term policy framework before submitting a formal request. A successor arrangement would support the authorities' efforts to tackle the aforementioned medium-term challenges and provide a safety net against possible shocks. It would likely be precautionary, but may have to be a drawing arrangement if the unidentified external financing envisaged in 2012-13, which could take the form of additional financing from the IFIs or higher private capital flows, is not mobilized as expected. The exact modalities of the arrangement will be discussed in the coming weeks.

\section{StAfF APPRAisal}

14. The SBA program objectives have been largely achieved, and Georgia is exiting from the current Fund arrangement in a relatively strong position. The economy is growing at a solid pace, enabling rapid fiscal consolidation; international reserves have been restored to the pre-crisis levels; and, with the normalization of financial sector conditions, bank lending has resumed at a healthy pace.

15. The successful Eurobond issue of April 2011 marks a significant improvement in market confidence and eases considerably debt rollover risks. The substantial downward repricing of Georgia risk embedded in the Eurobond issue is all the more remarkable as it came at a time of unsettled market conditions for sovereign paper.

16. While external financing and growth prospects have improved, the adjustment process has not been fully completed. Furthermore, the rise of external repayment obligations in 2012-13 creates vulnerabilities, owing to the uncertain external environment and possible political uncertainty around the elections of 2012 and 2013. The authorities' plan to mitigate such vulnerabilities by targeting a higher accumulation of international reserves in 2011 is welcome. 
17. The authorities' revised fiscal plan for 2011 targets a suitably ambitious adjustment. While remaining consistent with the frontloaded fiscal consolidation objectives anchoring the program, they also allow for an increase in social spending aimed at alleviating the social impact of higher food and fuel prices.

18. Sufficient flexibility to take revenue-enhancing measures should be preserved. The authorities should consider and be able to take such measures if faced with adverse fiscal shocks and/or difficulties in implementing their expenditure-based fiscal consolidation strategy in the run-up to the 2012-13 elections.

19. The monetary policy stance remains appropriate, given the absence of any evidence of second-round effects from the food and fuel price hikes. The authorities' readiness to adjust the policy stance if signs of more persistent inflation emerge is welcome.

20. The exchange rate has become demonstrably more flexible over the course of the SBA. Over the medium term, NBG's participation in the foreign exchange market should be guided by the objective of increasing NIR. To the extent that the authorities consider that intervention is needed to smooth excess volatility, it will be important that they build an extra buffer over and beyond their NIR targets to achieve this objective.

21. The banking sector is back on a relatively solid footing and banking supervision is moving appropriately to monitor the emergence of new vulnerabilities. The supervisory authority has made considerable strides to strengthen its internal analytical capacity and responsiveness to emerging risks. Stocktaking of the regulatory and supervision frameworks would be helpful at this stage to identify potential gaps.

22. Remaining challenges need to be addressed through a consistent set of fiscal, financial, and structural policies. Fund staff stands ready to assist the authorities in the implementation of such a strategy.

23. In light of the authorities' commitment to reduce the 2011 fiscal deficit by more than envisaged at the time of the seventh and eighth reviews, and thereby deliver the structural fiscal adjustment targeted under the program, staff supports the authorities' requests for completing the ninth Review and for a waiver of nonobservance of one performance criterion. 
Table 1. Georgia: Selected Macroeconomic Indicators, 2008-16

\begin{tabular}{|c|c|c|c|c|c|c|c|c|c|}
\hline & $\begin{array}{r}2008 \\
\text { Act. }\end{array}$ & $\begin{array}{r}2009 \\
\text { Act. }\end{array}$ & $\begin{array}{r}2010 \\
\text { Act. }\end{array}$ & $\begin{array}{r}2011 \\
\text { Proj. }\end{array}$ & $\begin{array}{c}2012 \\
\text { Proj. }\end{array}$ & $\begin{array}{c}2013 \\
\text { Proj. }\end{array}$ & $\begin{array}{r}2014 \\
\text { Proj. }\end{array}$ & $\begin{array}{r}2015 \\
\text { Proj. }\end{array}$ & $\begin{array}{r}2016 \\
\text { Proj. }\end{array}$ \\
\hline & \multicolumn{9}{|c|}{ (Annual percentage change, unless otherwise indicated) } \\
\hline \multicolumn{10}{|l|}{ National accounts } \\
\hline Nominal GDP (in million lari) & 19,075 & 17,986 & 20,791 & 23,577 & 26,428 & 29,345 & 32,430 & 35,839 & 39,607 \\
\hline Real GDP growth & 2.4 & -3.8 & 6.4 & 5.5 & 5.3 & 5.0 & 5.0 & 5.0 & 5.0 \\
\hline Population (in million) $1 /$ & 4.4 & 4.4 & 4.4 & 4.4 & 4.4 & 4.4 & 4.4 & 4.4 & 4.4 \\
\hline GDP deflator, period average & 9.6 & -2.0 & 8.7 & 7.5 & 6.5 & 5.8 & 5.3 & 5.3 & 5.3 \\
\hline Consumer price index, period average & 10.0 & 1.7 & 7.1 & 11.6 & 7.2 & 6.0 & 5.8 & 5.5 & 5.4 \\
\hline Consumer price index, end-of-period & 5.5 & 3.0 & 11.2 & 8.5 & 6.0 & 6.0 & 5.5 & 5.5 & 5.3 \\
\hline GDP per capita (in US\$) & 2,937 & 2,455 & 2,658 & 3,131 & 3,376 & 3,464 & 3,606 & 3,812 & 4,058 \\
\hline \multirow[t]{2}{*}{ Unemployment rate (in percent) } & 16.5 & 16.9 & $\cdots$ & $\cdots$ & $\cdots$ & $\cdots$ & $\cdots$ & $\cdots$ & $\cdots$ \\
\hline & \multicolumn{9}{|c|}{ (In percent of GDP) } \\
\hline \multicolumn{10}{|l|}{ Investment and saving } \\
\hline Public & 4.3 & 6.9 & 7.1 & 6.5 & 5.7 & 5.8 & 5.4 & 5.6 & 5.4 \\
\hline Private & 17.2 & 8.5 & 7.9 & 10.0 & 11.3 & 11.7 & 12.6 & 12.4 & 12.6 \\
\hline Gross national saving & -1.1 & 4.1 & 5.4 & 5.7 & 7.3 & 9.4 & 11.2 & 12.2 & 12.2 \\
\hline Public & 2.2 & -0.8 & 2.2 & 4.4 & 3.4 & 3.6 & 3.9 & 3.9 & 3.9 \\
\hline Private & -3.4 & 4.9 & 3.2 & 1.4 & 3.9 & 5.8 & 7.3 & 8.3 & 8.3 \\
\hline Saving-investment balance & -22.6 & -11.2 & -9.6 & -10.8 & -9.7 & -8.1 & -6.8 & -5.8 & -5.8 \\
\hline \multicolumn{10}{|l|}{ Consolidated government operations } \\
\hline Total government debt & 27.6 & 37.3 & 39.1 & 36.8 & 37.6 & 37.0 & 35.5 & 34.8 & 34.0 \\
\hline Of which: foreign-currency denominated & 23.5 & 31.7 & 33.6 & 31.3 & 31.7 & 31.2 & 29.8 & 29.3 & 28.7 \\
\hline Revenue 3/ & 30.7 & 29.3 & 28.2 & 28.5 & 26.5 & 26.3 & 26.1 & 26.1 & 26.1 \\
\hline Current expenditures & 28.5 & 30.1 & 26.0 & 24.1 & 23.0 & 22.7 & 22.2 & 22.2 & 22.2 \\
\hline Operating balance & 2.2 & -0.8 & 2.2 & 4.4 & 3.4 & 3.6 & 3.9 & 3.9 & 3.9 \\
\hline Capital spending and net lending & 8.6 & 8.4 & 8.8 & 8.0 & 6.7 & 6.5 & 5.9 & 5.6 & 5.4 \\
\hline Domestic & -2.3 & 3.3 & 0.0 & -0.3 & 0.3 & 1.0 & 1.5 & 0.6 & 0.3 \\
\hline External & 5.0 & 3.9 & 5.5 & 3.0 & 1.9 & 1.1 & 0.4 & 1.1 & 1.2 \\
\hline Of which : unidentified & 0.0 & 0.0 & 0.0 & 0.0 & 0.0 & 0.7 & 0.0 & 0.0 & 0.0 \\
\hline Privatization receipts & 3.7 & 2.0 & 1.1 & 0.8 & 0.4 & 0.2 & 0.2 & 0.0 & 0.0 \\
\hline \multicolumn{10}{|c|}{ (Annual percentage change, unless otherwise indicated) } \\
\hline \multicolumn{10}{|l|}{ Monetary sector } \\
\hline Reserve money & -4.5 & 21.8 & 4.5 & 7.0 & 10.0 & 9.3 & 9.3 & 9.3 & 9.3 \\
\hline Broad money (including fx deposits) 4 / & 7.0 & 8.1 & 28.5 & 20.0 & 21.0 & 19.0 & 19.0 & 19.0 & 19.0 \\
\hline Bank credit to the private sector & 28.2 & -13.5 & 20.5 & 15.8 & 18.3 & 15.0 & 13.8 & 13.4 & 18.5 \\
\hline Deposit interest rate (annual weighted average on flows) & 9.5 & 9.4 & 7.9 & $\ldots$ & $\ldots$ & $\ldots$ & $\ldots$ & $\ldots$ & $\ldots$ \\
\hline Lending interest rate (annual weighted average on flows) & 22.7 & 20.8 & 17.7 & $\cdots$ & $\cdots$ & $\cdots$ & $\ldots$ & $\cdots$ & $\cdots$ \\
\hline \multicolumn{10}{|l|}{ External sector } \\
\hline Exports of goods and services (percent of GDP) & 28.7 & 29.8 & 34.8 & 36.7 & 37.4 & 38.6 & 38.8 & 38.9 & 39.1 \\
\hline Annual percentage change & 15.9 & -13.0 & 26.6 & 24.2 & 10.0 & 6.0 & 4.7 & 6.2 & 7.0 \\
\hline Imports of goods and services (percent of GDP) & 58.3 & 48.9 & 52.2 & 53.8 & 52.5 & 51.8 & 51.0 & 50.2 & 50.5 \\
\hline Annual percentage change & 26.8 & -29.8 & 15.7 & 21.5 & 5.2 & 1.4 & 2.5 & 4.1 & 7.2 \\
\hline Net imports of oil (in US\$) & 762 & 555 & 638 & 849 & 886 & 898 & 931 & 980 & 1039 \\
\hline Current account balance (in millions of US\$) & $-2,912$ & $-1,210$ & $-1,117$ & $-1,481$ & $-1,439$ & $-1,228$ & $-1,085$ & -970 & $-1,039$ \\
\hline In percent of GDP & -22.6 & -11.2 & -9.6 & -10.8 & -9.7 & -8.1 & -6.8 & -5.8 & -5.8 \\
\hline Gross international reserves (in millions of US\$) & 1,480 & 2,111 & 2,263 & 2,795 & 2,669 & 2,669 & 2,715 & 3,005 & 3,097 \\
\hline In months of next year's imports of goods and services & 3.4 & 4.2 & 3.7 & 4.3 & 4.1 & 4.0 & 3.9 & 4.0 & 3.9 \\
\hline Foreign direct investment (percent of GDP) & 12.2 & 6.1 & 4.3 & 5.2 & 5.4 & 5.7 & 5.5 & 5.5 & 5.5 \\
\hline Average exchange rate (lari per US\$) & 1.48 & 1.67 & 1.78 & $\ldots$ & $\ldots$ & $\ldots$ & $\ldots$ & $\ldots$ & $\ldots$ \\
\hline
\end{tabular}

Sources: Georgian authorities; and Fund staff estimates.

1/ Excludes Abkhazia residents.

2/ Investment is measured on a net basis (acquisitions minus disposals of nonfinancial assets).

3/ Includes grants.

4/ The proceeds of the Georgian Railway eurobond issuance from July 2010, which were deposited in accounts with Georgian commercial banks that placed them abroad are not included in broad money. 
Table 2. Georgia: General Government Operations, 2010-16 1/

\begin{tabular}{|c|c|c|c|c|c|c|c|c|c|c|c|c|c|c|c|c|c|c|}
\hline & \multirow[t]{2}{*}{2010} & \multicolumn{2}{|c|}{2011 Q1 } & \multicolumn{2}{|c|}{2011 annual } & \multirow{2}{*}{$\begin{array}{l}2012 \\
\text { Proj. }\end{array}$} & \multirow{2}{*}{$\begin{array}{l}2013 \\
\text { Proj. }\end{array}$} & \multirow{2}{*}{$\begin{array}{l}2014 \\
\text { Proj. }\end{array}$} & \multirow{2}{*}{$\begin{array}{l}2015 \\
\text { Proj. }\end{array}$} & \multirow{2}{*}{$\begin{array}{l}2016 \\
\text { Proj. }\end{array}$} & \multirow[t]{2}{*}{2010} & \multicolumn{2}{|c|}{2011} & \multirow{2}{*}{$\begin{array}{l}2012 \\
\text { Proj. }\end{array}$} & \multirow{2}{*}{$\begin{array}{l}2013 \\
\text { Proj. }\end{array}$} & \multirow{2}{*}{$\begin{array}{l}2014 \\
\text { Proj. }\end{array}$} & \multirow{2}{*}{$\begin{array}{l}2015 \\
\text { Proj. }\end{array}$} & \multirow{2}{*}{$\begin{array}{l}2016 \\
\text { Proj. }\end{array}$} \\
\hline & & Proj. & Act. & 7/8 Rev. & Proj. & & & & & & & 7/8 Rev. & Proj. & & & & & \\
\hline & \multicolumn{10}{|c|}{ (In millions of lari) } & \multicolumn{8}{|c|}{ (In percent of GDP) } \\
\hline Revenues & 5,866 & 1,383 & 1,765 & 6,315 & 6,713 & 6,998 & 7,718 & 8,470 & 9,350 & 10,321 & 28.2 & 27.4 & 28.5 & 26.5 & 26.3 & 26.1 & 26.1 & 26.1 \\
\hline Taxes & 4,867 & 1,247 & 1,559 & 5,571 & 5,879 & 6,332 & 7,031 & 7,770 & 8,587 & 9,489 & 23.4 & 24.2 & 24.9 & 24.0 & 24.0 & 24.0 & 24.0 & 24.0 \\
\hline Other revenues & 526 & 71 & 127 & 440 & 485 & 515 & 543 & 567 & 627 & 693 & 2.5 & 1.9 & 2.1 & 1.9 & 1.8 & 1.7 & 1.7 & 1.7 \\
\hline Grants & 473 & 65 & 79 & 304 & 349 & 151.4 & 144 & 133 & 136 & 139 & 2.3 & 1.3 & 1.5 & 0.6 & 0.5 & 0.4 & 0.4 & 0.4 \\
\hline Current expenditures & 5,399 & 1,254 & 1,241 & 5,550 & 5,681 & 6,091 & 6,650 & 7,211 & 7,959 & 8,785 & 26.0 & 24.1 & 24.1 & 23.0 & 22.7 & 22.2 & 22.2 & 22.2 \\
\hline Compensation of employees & 1,138 & 270 & 265 & 1,208 & 1,150 & 1,251 & 1,390 & 1,460 & 1,613 & 1,783 & 5.5 & 5.2 & 4.9 & 4.7 & 4.7 & 4.5 & 4.5 & 4.5 \\
\hline Use of goods and services & 1,086 & 202 & 239 & 1,016 & 1,077 & 1,150 & 1,250 & 1,350 & 1,492 & 1,649 & 5.2 & 4.4 & 4.6 & 4.4 & 4.3 & 4.2 & 4.2 & 4.2 \\
\hline Subsidies & 372 & 90 & 94 & 380 & 381 & 380 & 390 & 400 & 442 & 489 & 1.8 & 1.6 & 1.6 & 1.4 & 1.3 & 1.2 & 1.2 & 1.2 \\
\hline Grants & 13 & 3 & 6 & 12 & 14 & 15 & 17 & 20 & 22 & 24 & 0.1 & 0.1 & 0.1 & 0.1 & 0.1 & 0.1 & 0.1 & 0.1 \\
\hline Social expenses & 1,612 & 408 & 412 & 1,631 & 1,700 & 1,940 & 2,130 & 2,350 & 2,597 & 2,870 & 7.8 & 7.1 & 7.2 & 7.3 & 7.3 & 7.2 & 7.2 & 7.2 \\
\hline Other expenses & 972 & 225 & 182 & 1,013 & 1,037 & 1,050 & 1,100 & 1,230 & 1,359 & 1,502 & 4.7 & 4.4 & 4.4 & 4.0 & 3.7 & 3.8 & 3.8 & 3.8 \\
\hline Interest & 206 & 56 & 43 & 292 & 322 & 305 & 373 & 401 & 433 & 468 & 1.0 & 1.3 & 1.4 & 1.2 & 1.3 & 1.2 & 1.2 & 1.2 \\
\hline To nonresidents & 133 & 33 & 19 & 191 & 217 & 186 & 234 & 249 & 268 & 291 & 0.6 & 0.8 & 0.9 & 0.7 & 0.8 & 0.8 & 0.7 & 0.7 \\
\hline To residents & 74 & 23 & 25 & 101 & 105 & 118 & 138 & 152 & 164 & 176 & 0.4 & 0.4 & 0.4 & 0.4 & 0.5 & 0.5 & 0.5 & 0.4 \\
\hline Operating balance & 467 & 129 & 524 & 765 & 1,032 & 908 & 1,068 & 1,259 & 1,391 & 1,536 & 2.2 & 3.3 & 4.4 & 3.4 & 3.6 & 3.9 & 3.9 & 3.9 \\
\hline Capital spending and net lending & 1,834 & 299 & 327 & 1,748 & 1,881 & 1,780 & 1,919 & 1,908 & 2,000 & 2,137 & 8.8 & 7.6 & 8.0 & 6.7 & 6.5 & 5.9 & 5.6 & 5.4 \\
\hline Capital & 1,706 & 256 & 309 & 1,630 & 1,724 & 1,604 & 1,747 & 1,812 & 1,960 & 2,137 & 8.2 & 7.1 & 7.3 & 6.1 & 6.0 & 5.6 & 5.5 & 5.4 \\
\hline Net lending & 128 & 43 & 19 & 118 & 157 & 176 & 172 & 96 & 40 & 0 & 0.6 & 0.5 & 0.7 & 0.7 & 0.6 & 0.3 & 0.1 & 0.0 \\
\hline Overall balance & $-1,366$ & -170 & 197 & -983 & -849 & -872 & -851 & -649 & -609 & -594 & -6.6 & -4.3 & -3.6 & -3.3 & -2.9 & -2.0 & -1.7 & -1.5 \\
\hline Statistical discrepancy & 0 & 0 & 0 & 0 & 0 & 0 & 0 & 0 & 0 & 0 & 0.0 & 0.0 & 0.0 & 0.0 & 0.0 & 0.0 & 0.0 & 0.0 \\
\hline Total financing & 1,366 & 170 & -197 & 983 & 849 & 694 & 466 & 649 & 609 & 594 & 6.6 & 4.3 & 3.6 & 2.6 & 1.6 & 2.0 & 1.7 & 1.5 \\
\hline Domestic & -6 & 73 & -289 & 209 & -67 & 90 & 280 & 472 & 227 & 120 & 0.0 & 0.9 & -0.3 & 0.3 & 1.0 & 1.5 & 0.6 & 0.3 \\
\hline Net T-bill issuance & 172 & 9 & 12 & 100 & 200 & 300 & 175 & 175 & 150 & 150 & 0.8 & 0.4 & 0.8 & 1.1 & 0.6 & 0.5 & 0.4 & 0.4 \\
\hline Amortization 2/ & -42 & -5 & -7 & -35 & -35 & -35 & -35 & -35 & -35 & -35 & -0.2 & -0.2 & -0.1 & -0.1 & -0.1 & -0.1 & -0.1 & -0.1 \\
\hline Use of deposits at the NBG and banks & -136 & 69 & -294 & 144 & -232 & -175 & 140 & 332 & 112 & 5 & -0.7 & 0.6 & -1.0 & -0.7 & 0.5 & 1.0 & 0.3 & 0.0 \\
\hline External & 1,152 & 71 & 52 & 624 & 716 & 504 & 136 & 127 & 382 & 474 & 5.5 & 2.7 & 3.0 & 1.9 & 0.5 & 0.4 & 1.1 & 1.2 \\
\hline Borrowing & 1,275 & 94 & 71 & 761 & 1,577 & 614 & 661 & 687 & 723 & 755 & 6.1 & 3.3 & 6.7 & 2.3 & 2.3 & 2.1 & 2.0 & 1.9 \\
\hline of which: IMF & 523 & 0 & 0 & 0 & 0 & 0 & 0 & 0 & 0 & 0 & 2.5 & 0.0 & 0.0 & 0.0 & 0.0 & 0.0 & 0.0 & 0.0 \\
\hline Amortization & -123 & -23 & -19 & -137 & -861 & -110 & -525 & -561 & -341 & -281 & -0.6 & -0.6 & -3.7 & -0.4 & -1.8 & -1.7 & -1.0 & -0.7 \\
\hline of which: IMF & 0 & 0 & 0 & 0 & 0 & -22 & -283 & -395 & -111 & 0 & 0.0 & 0.0 & 0.0 & -0.1 & -1.0 & -1.2 & -0.3 & 0.0 \\
\hline Use of Sovereign Wealth Fund resources & 0 & 0 & 0 & 0 & 0 & 0 & 0 & 0 & 0 & 0 & 0.0 & 0.0 & 0.0 & 0.0 & 0.0 & 0.0 & 0.0 & 0.0 \\
\hline Privatization receipts & 220 & 26 & 40 & 150 & 200 & 100 & 50 & 50 & 0 & 0 & 1.1 & 0.7 & 0.8 & 0.4 & 0.2 & 0.2 & 0.0 & 0.0 \\
\hline Unidentified external financing & 0 & 0 & 0 & 0 & 0 & 178 & 385 & 0 & 0 & 0 & 0.0 & 0.0 & 0.0 & 0.7 & 1.3 & 0.0 & 0.0 & 0.0 \\
\hline \multicolumn{19}{|l|}{ Memorandum items: } \\
\hline Nominal GDP & 20,791 & 4,831 & 4,987 & 23,018 & 23,577 & 26,428 & 29,345 & 32,430 & 35,839 & 39,607 & & & & & & & & \\
\hline Fiscal deficit excluding grants & 1,839 & & & 1,287 & 1,198 & 845 & 610 & 781 & 745 & 733 & 8.8 & 5.6 & 5.1 & 3.2 & 2.1 & 2.4 & 2.1 & 1.9 \\
\hline Total expenditures (current prices) 3/ & 7,232 & 1,553 & 1,568 & 7,298 & 7,561 & 7,870 & 8,568 & 9,118 & 9,959 & 10,921 & 34.8 & 31.7 & 32.1 & 29.8 & 29.2 & 28.1 & 27.8 & 27.6 \\
\hline Total expenditures (constant 2008 prices) & 6,790 & & & 6,461 & 6,604 & 6,454 & 6,645 & 6,719 & 6,972 & 7,264 & & & & & & & & \\
\hline
\end{tabular}

CInternational Monetary Fund. Not for Redistribution 
Table 3. Georgia: Summary Balance of Payments, 2008-16

(In millions of U.S. dollars)

\begin{tabular}{|c|c|c|c|c|c|c|c|c|c|c|c|}
\hline & \multirow[t]{3}{*}{2008} & \multirow[t]{3}{*}{2009} & \multicolumn{2}{|c|}{2010} & \multicolumn{2}{|c|}{2011} & \multirow{3}{*}{$\begin{array}{l}2012 \\
\text { Proj. }\end{array}$} & \multirow{3}{*}{$\begin{array}{l}2013 \\
\text { Proj. }\end{array}$} & \multirow{3}{*}{$\begin{array}{l}2014 \\
\text { Proj. }\end{array}$} & \multirow{3}{*}{$\begin{array}{l}2015 \\
\text { Proj. }\end{array}$} & \multirow{3}{*}{$\begin{array}{l}2016 \\
\text { Proj. }\end{array}$} \\
\hline & & & 7th-8th & & 7th-8th & & & & & & \\
\hline & & & Review & Act. & Review & Proj. & & & & & \\
\hline Current account balance & $-2,912$ & $-1,210$ & $-1,329$ & $-1,117$ & $-1,587$ & $-1,481$ & $-1,439$ & $-1,228$ & $-1,085$ & -970 & $-1,039$ \\
\hline Trade balance & $-3,833$ & $-2,399$ & $-2,630$ & $-2,567$ & $-2,847$ & $-3,129$ & $-3,158$ & $-3,045$ & $-3,019$ & $-3,044$ & $-3,225$ \\
\hline Exports & 2,428 & 1,894 & 2,339 & 2,460 & 2,592 & 3,008 & 3,296 & 3,478 & 3,629 & 3,865 & 4,162 \\
\hline Imports & $-6,261$ & $-4,293$ & $-4,969$ & $-5,027$ & $-5,439$ & $-6,137$ & $-6,454$ & $-6,522$ & $-6,648$ & $-6,908$ & $-7,386$ \\
\hline Services & 21 & 340 & 402 & 534 & 501 & 772 & 917 & 1,024 & 1,078 & 1,148 & 1,183 \\
\hline Services: credit & 1,260 & 1,314 & 1,455 & 1,602 & 1,638 & 2,036 & 2,250 & 2,399 & 2,524 & 2,668 & 2,828 \\
\hline Services: debit & $-1,239$ & -974 & $-1,053$ & $-1,067$ & $-1,137$ & $-1,264$ & $-1,333$ & $-1,375$ & $-1,445$ & $-1,520$ & $-1,645$ \\
\hline Income (net) & -161 & -118 & -177 & -183 & -282 & -300 & -347 & -372 & -358 & -336 & -306 \\
\hline Of which: interest payments & -249 & -247 & -311 & -265 & -383 & -364 & -418 & -444 & -436 & -432 & -399 \\
\hline Transfers (net) & 1,060 & 967 & 1,075 & 1,099 & 1,041 & 1,176 & 1,149 & 1,165 & 1,214 & 1,262 & 1,309 \\
\hline Of which: public sector & 365 & 140 & 187 & 175 & 128 & 149 & 80 & 54 & 53 & 53 & 52 \\
\hline Capital account & 112 & 183 & 221 & 206 & 116 & 137 & 91 & 83 & 74 & 75 & 74 \\
\hline General government & 87 & 170 & 203 & 189 & 91 & 117 & 60 & 50 & 40 & 39 & 38 \\
\hline Other sectors & 25 & 13 & 18 & 16 & 25 & 20 & 31 & 33 & 34 & 36 & 36 \\
\hline Financial account & 2,784 & 1,428 & 923 & 793 & 1,684 & 1,935 & 1,372 & 1,337 & 1,316 & 1,267 & 1,073 \\
\hline Direct investment (net) & 1,494 & 659 & 603 & 493 & 835 & 712 & 806 & 865 & 877 & 928 & 989 \\
\hline Monetary authorities, net $1 /$ & 22 & 247 & 0 & -1 & 0 & 0 & 0 & 0 & 0 & 0 & 0 \\
\hline General government & 651 & 386 & 367 & 335 & 431 & 386 & 296 & 218 & 255 & 231 & 214 \\
\hline Portfolio investment (net) & 501 & 0 & 0 & 0 & 0 & 65 & 0 & -65 & 0 & 0 & 0 \\
\hline Long-term loans received & 191 & 359 & 366 & 334 & 431 & 352 & 296 & 283 & 255 & 231 & 214 \\
\hline Drawing & 227 & 428 & 440 & 401 & 506 & 421 & 345 & 343 & 336 & 339 & 341 \\
\hline Repayment & -36 & -69 & -74 & -67 & -75 & -69 & -49 & -61 & -81 & -108 & -127 \\
\hline Other, net & -41 & 27 & 1 & 1 & 0 & -31 & 0 & 0 & 0 & 0 & 0 \\
\hline Private Sector, excl. FDI & 617 & 136 & -46 & -34 & 417 & 837 & 270 & 254 & 184 & 108 & -130 \\
\hline Banks & 403 & -65 & -175 & -217 & 315 & 563 & 125 & 200 & 95 & 104 & 70 \\
\hline Portfolio investment, net & 109 & 8 & 52 & 5 & 5 & 5 & 2 & 13 & 12 & 12 & 13 \\
\hline Of which: equity liabilities & 101 & 8 & 52 & -7 & 5 & 5 & 7 & 10 & 12 & 13 & 14 \\
\hline Loans received (net) & 596 & -243 & -51 & 6 & -110 & 235 & 2 & 214 & 66 & 98 & 73 \\
\hline Other, net (currency and deposits) & -302 & 171 & -176 & -228 & 420 & 322 & 122 & -27 & 17 & -6 & -16 \\
\hline Other sectors & 214 & 201 & 129 & 183 & 102 & 274 & 145 & 54 & 88 & 4 & -200 \\
\hline Portfolio investment, net & 17 & 4 & 247 & 262 & 0 & 0 & 0 & 5 & 25 & 27 & -221 \\
\hline Long-term loans received (net) & 61 & 153 & -91 & -50 & 46 & 146 & 161 & 114 & 18 & -40 & -26 \\
\hline Other, net & 137 & 44 & -27 & -29 & 56 & 128 & -16 & -65 & 46 & 17 & 47 \\
\hline Errors and omissions & -36 & 51 & 72 & 87 & 0 & 0 & 0 & 0 & 0 & 0 & 0 \\
\hline Overall balance & -52 & 451 & -113 & -30 & 213 & 591 & 23 & 192 & 306 & 371 & 108 \\
\hline Financing & 52 & -451 & 113 & 30 & -213 & -591 & -123 & -392 & -306 & -371 & -108 \\
\hline Gross International Reserves (-increase) & -131 & -616 & -158 & -208 & -413 & -531 & 126 & 0 & -46 & -290 & -93 \\
\hline Use of Fund Resources & 222 & 313 & 277 & 276 & 200 & -60 & -250 & -392 & -260 & -81 & -15 \\
\hline Purchases (SBA) & 257 & 340 & 298 & 297 & 258 & 0 & 0 & 0 & 0 & 0 & 0 \\
\hline Of which: augmentation & & & 303 & 305 & 258 & 0 & & & $\ldots$ & $\ldots$ & $\cdots$ \\
\hline Repayments (SBA and ECF 2/) & -35 & -28 & -21 & -22 & -58 & -60 & -250 & -392 & -260 & -81 & -15 \\
\hline Exceptional financing & -39 & -148 & -7 & -37 & 0 & 0 & 0 & 0 & 0 & 0 & 0 \\
\hline Unidentified external financing (+ overfinancing) & 0 & 0 & 0 & 0 & 0 & 0 & -100 & -200 & 0 & 0 & 0 \\
\hline Memorandum items: & & & & & & & & & & & \\
\hline Nominal GDP & 12,870 & 10,768 & 11,491 & 11,665 & 12,647 & 13,754 & 14,840 & 15,236 & 15,874 & 16,792 & 17,890 \\
\hline Current account balance (percent of GDP) & -22.6 & -11.2 & -11.6 & -9.6 & -12.6 & -10.8 & -9.7 & -8.1 & -6.8 & -5.8 & -5.8 \\
\hline excluding official transfers (percent of GDP) & -25.5 & -12.5 & -13.2 & -11.1 & -13.6 & -11.8 & -10.2 & -8.4 & -7.2 & -6.1 & -6.1 \\
\hline Trade balance (in percent of GDP) & -29.8 & -22.3 & -22.9 & -22.0 & -22.5 & -22.7 & -21.3 & -20.0 & -19.0 & -18.1 & -18.0 \\
\hline GNFS exports growth (percent) & 15.9 & -13.0 & 18.5 & 26.6 & 11.5 & 24.2 & 10.0 & 6.0 & 4.7 & 6.2 & 7.0 \\
\hline GNFS exports volume growth (percent) & -1.4 & -0.6 & 5.0 & 6.4 & 8.7 & 5.8 & 9.3 & 8.6 & 6.8 & 6.2 & 5.4 \\
\hline GNFS imports growth (percent) & 26.8 & -29.8 & 14.3 & 15.7 & 9.2 & 21.5 & 5.2 & 1.4 & 2.5 & 4.1 & 7.2 \\
\hline GNFS imports volume growth (percent) & 8.3 & -18.6 & 3.6 & 0.6 & 7.5 & 3.1 & 5.5 & 4.0 & 5.1 & 5.4 & 7.0 \\
\hline Net capital inflows to private sector & 2,111 & 796 & 556 & 459 & 1,253 & 1,549 & 1,076 & 1,120 & 1,061 & 1,036 & 859 \\
\hline (in percent of GDP) & 16.4 & 7.4 & 4.8 & 3.9 & 9.9 & 11.3 & 7.2 & 7.3 & 6.7 & 6.2 & 4.8 \\
\hline Gross international reserves (end of period) & 1,480 & 2,111 & 2,202 & 2,263 & 2,615 & 2,795 & 2,669 & 2,669 & 2,715 & 3,005 & 3,097 \\
\hline (in months of next year GNFS imports) & 3.4 & 4.2 & 4.0 & 3.7 & 4.6 & 4.3 & 4.1 & 4.0 & 3.9 & 4.0 & 3.9 \\
\hline External debt (nominal) & 5,664 & 6,250 & 7,397 & 7,825 & 8,238 & 8,524 & 8,891 & 8,922 & 8,717 & 8,814 & 8,680 \\
\hline (in percent of GDP) & 44.0 & 58.0 & 64.4 & 67.1 & 65.1 & 62.0 & 59.9 & 58.6 & 54.9 & 52.5 & 48.5 \\
\hline MLT External debt service & 591 & 758 & 758 & 698 & 881 & 1,356 & 1,327 & 1,367 & 1,227 & 1,121 & 1,070 \\
\hline (in percent of exports) & 16.0 & 23.6 & 20.0 & 17.2 & 20.8 & 26.9 & 23.9 & 23.3 & 19.9 & 17.2 & 15.3 \\
\hline External public sector debt (nominal) & 2,691 & 3,382 & 4,186 & 3,937 & 4,855 & 4,323 & 4,513 & 4,581 & 4,620 & 4,816 & 5,060 \\
\hline (in percent of GDP) & 20.9 & 31.4 & 36.4 & 33.7 & 38.4 & 31.4 & 30.4 & 30.1 & 29.1 & 28.7 & 28.3 \\
\hline External public debt service & 126 & 168 & 193 & 168 & 252 & 663 & 414 & 647 & 476 & 335 & 303 \\
\hline (in percent of exports) & 3.4 & 5.2 & 5.1 & 4.1 & 6.0 & 13.1 & 7.5 & 11.0 & 7.7 & 5.1 & 4.3 \\
\hline
\end{tabular}

Sources: National Bank of Georgia, Ministry of Finance, and Fund staff estimates.

1/ SDR allocation included under monetary authorities' long-term liabilities.

2/ Following the Low Income Countries (LIC) reforms, effective January 7 2010, the PRGF arrangements were renamed Extended Credit Facility (ECF) arrangements. 
Table 4. Georgia: Accounts of the National Bank of Georgia, 2010-16

\begin{tabular}{|c|c|c|c|c|c|c|c|c|c|c|c|c|c|}
\hline & \multicolumn{4}{|c|}{2010} & \multicolumn{4}{|c|}{2011} & \multirow{2}{*}{$\begin{array}{l}2012 \\
\text { Proj. }\end{array}$} & \multirow{2}{*}{$\begin{array}{l}2013 \\
\text { Proj. }\end{array}$} & \multirow{2}{*}{$\begin{array}{l}2014 \\
\text { Proj. }\end{array}$} & \multirow{2}{*}{$\begin{array}{l}2015 \\
\text { Proj. }\end{array}$} & \multirow{2}{*}{$\begin{array}{l}2016 \\
\text { Proj. }\end{array}$} \\
\hline & $\begin{array}{l}\text { Mar. } \\
\text { Act. }\end{array}$ & $\begin{array}{l}\text { Jun. } \\
\text { Act. }\end{array}$ & $\begin{array}{l}\text { Sep. } \\
\text { Act. }\end{array}$ & $\begin{array}{l}\text { Dec. } \\
\text { Act. }\end{array}$ & $\begin{array}{l}\text { Mar. } \\
\text { Act. }\end{array}$ & $\begin{array}{l}\text { Jun. } \\
\text { Proj. }\end{array}$ & $\begin{array}{l}\text { Sep. } \\
\text { Proj. }\end{array}$ & $\begin{array}{l}\text { Dec. } \\
\text { Proj }\end{array}$ & & & & & \\
\hline & \multicolumn{13}{|c|}{ (In millions of lari) } \\
\hline Net foreign exchange position & 2,117 & 1,698 & 1,961 & 2,218 & 2,354 & 2,470 & 2,479 & 2,469 & 2,796 & 3,331 & 3,565 & 4,214 & 4,375 \\
\hline Gross International Reserves & 3,847 & 3,438 & 3,812 & 4,013 & 4,636 & 4,773 & 4,794 & 4,768 & 4,952 & 5,327 & 5,673 & 6,546 & 6,966 \\
\hline Other foreign assets & 6 & 5 & 5 & 8 & 3 & 3 & 3 & 7 & 8 & 9 & 9 & 9 & 10 \\
\hline Foreign currency liabilities & $-1,736$ & $-1,746$ & $-1,856$ & $-1,803$ & $-2,285$ & $-2,307$ & $-2,319$ & $-2,307$ & $-2,164$ & $-2,004$ & $-2,117$ & $-2,342$ & $-2,601$ \\
\hline Of which: use of Fund resources & $-1,143$ & $-1,165$ & $-1,186$ & $-1,150$ & $-1,116$ & $-1,106$ & $-1,093$ & $-1,027$ & -674 & -236 & -107 & -47 & -15 \\
\hline Of which: compulsory reserves in USD & -142 & -148 & -242 & -245 & -771 & -806 & -831 & -885 & $-1,062$ & $-1,309$ & $-1,528$ & $-1,793$ & $-2,070$ \\
\hline Of which: swap liabilities & -63 & -33 & 0 & 0 & 0 & 0 & 0 & 0 & 0 & 0 & 0 & 0 & 0 \\
\hline Net domestic assets & -484 & -37 & -265 & -396 & -821 & -693 & -664 & -519 & -651 & -987 & $-1,003$ & $-1,414$ & $-1,315$ \\
\hline Net claims on general government & -194 & 52 & -73 & -95 & -368 & -258 & -266 & -305 & -515 & -410 & -113 & -36 & -66 \\
\hline Claims on general government (incl. T-bills) & 746 & 733 & 718 & 714 & 709 & 704 & 692 & 682 & 647 & 612 & 577 & 542 & 507 \\
\hline Nontradable govt. debt & 601 & 601 & 601 & 601 & 561 & 561 & 561 & 561 & 521 & 481 & 441 & 401 & 361 \\
\hline Securitized debt (marketable) & 146 & 132 & 117 & 113 & 148 & 143 & 131 & 121 & 126 & 131 & 136 & 141 & 146 \\
\hline Deposits & -940 & -681 & -790 & -809 & $-1,078$ & -962 & -958 & -987 & $-1,162$ & $-1,022$ & -690 & -578 & -573 \\
\hline Claims on rest of economy & 3 & 3 & 2 & 2 & 2 & 2 & 2 & 2 & 2 & 2 & 2 & 2 & 2 \\
\hline Claims on banks & -89 & 57 & -7 & -165 & -354 & -336 & -300 & -115 & 208 & -8 & -170 & -505 & -248 \\
\hline Bank refinancing & 45 & 217 & 185 & 190 & 95 & 95 & 95 & 95 & 308 & 95 & 95 & 95 & 95 \\
\hline Certificates of deposits and bonds & -194 & -190 & -192 & -355 & -449 & -431 & -395 & -210 & -100 & -103 & -265 & -600 & -343 \\
\hline Other items, net & -204 & -149 & -188 & -138 & -100 & -100 & -100 & -100 & -346 & -571 & -722 & -874 & $-1,003$ \\
\hline Reserve money & 1,633 & 1,661 & 1,696 & 1,822 & 1,533 & $1,776.9$ & $1,814.2$ & $1,949.8$ & 2,145 & 2,344 & 2,562 & 2,801 & 3,060 \\
\hline Currency in circulation & 1,399 & 1,460 & 1,501 & 1,618 & 1,395 & 1,625 & 1,650 & 1,775 & 1,925 & 2,075 & 2,225 & 2,375 & 2,525 \\
\hline Bank lari reserves $1 /$ & 235 & 127 & 150 & 144 & 128 & 140 & 151 & 168 & 210 & 257 & 328 & 419 & 534 \\
\hline \multirow[t]{2}{*}{ Overnight deposits } & 0 & 74 & 45 & 60 & 10 & 12 & 13 & 7 & 10 & 12 & 10 & 7 & 1 \\
\hline & \multicolumn{13}{|c|}{ (Percent contribution, compared to reserve money at the end of previous year) } \\
\hline Net foreign exchange position & 15.8 & -8.2 & 6.8 & 21.6 & 7.4 & 13.8 & 14.3 & 13.7 & 16.8 & 24.9 & 10.0 & 25.3 & 5.7 \\
\hline Net domestic assets & -22.2 & 3.5 & -9.6 & -17.1 & -23.3 & -16.3 & -14.7 & -6.7 & -6.8 & -15.6 & -0.7 & -16.0 & 3.5 \\
\hline Net claims on general government & -21.1 & -7.0 & -14.1 & -15.4 & -15.0 & -9.0 & -9.4 & -11.5 & -10.8 & 4.9 & 12.7 & 3.0 & -1.1 \\
\hline Claims on rest of economy & 0.0 & 0.0 & 0.0 & -0.1 & 0.0 & 0.0 & 0.0 & 0.0 & 0.0 & 0.0 & 0.0 & 0.0 & 0.0 \\
\hline Claims on banks & 1.4 & 9.8 & 6.1 & -2.9 & -10.4 & -9.4 & -7.4 & 2.7 & 16.6 & -10.1 & -6.9 & -13.1 & 9.2 \\
\hline \multirow[t]{2}{*}{ Other items, net } & -2.5 & 0.7 & -1.6 & 1.2 & 2.1 & 2.1 & 2.1 & 2.1 & -12.6 & -10.5 & -6.4 & -6.0 & -4.6 \\
\hline & \multicolumn{13}{|c|}{ (Percentage change, relative to end of previous year) } \\
\hline Reserve money & -6.3 & -4.8 & -2.8 & 4.5 & -15.9 & -2.5 & -0.4 & 7.0 & 10.0 & 9.3 & 9.3 & 9.3 & 9.3 \\
\hline Currency in circulation & -4.1 & 0.2 & 2.9 & 11.0 & -13.8 & 0.4 & 2.0 & 9.7 & 8.5 & 7.8 & 7.2 & 6.7 & 6.3 \\
\hline Bank lari reserves $1 /$ & -18.0 & -55.8 & -47.7 & -49.7 & -11.4 & -2.9 & 5.1 & 16.5 & 25.2 & 22.5 & 27.4 & 27.9 & 27.3 \\
\hline \multicolumn{14}{|l|}{ Memorandum items: } \\
\hline Net international reserves & & & & & & & & & & & & & \\
\hline (in millions of USD, at prog. exchange rates) $2 /$ & 897 & 647 & 708 & 882 & 994 & 1,063 & 1,085 & 1,090 & 1,161 & 1,472 & 1,693 & 1,952 & 1,927 \\
\hline Net domestic assets (in millions of lari, at prog. exchange rate) $2 /$ & 135 & 579 & 513 & 349 & -126 & 1 & 3 & 130 & 206 & -114 & -264 & -459 & -158 \\
\hline Reserve money (in percent, 12-month growth) & 28.9 & 8.6 & 0.3 & 4.5 & -6.1 & 5.0 & 9.2 & 7.0 & 10.0 & 9.3 & 9.3 & 9.3 & 9.3 \\
\hline
\end{tabular}

Sources: National Bank of Georgia; and Fund staff estimates.

$1 /$ Comprises required and excess reserves on lari-denominated deposits.

2/ Based on program definition as defined in the TMU. 
Table 5. Georgia: Monetary Survey, 2010-16

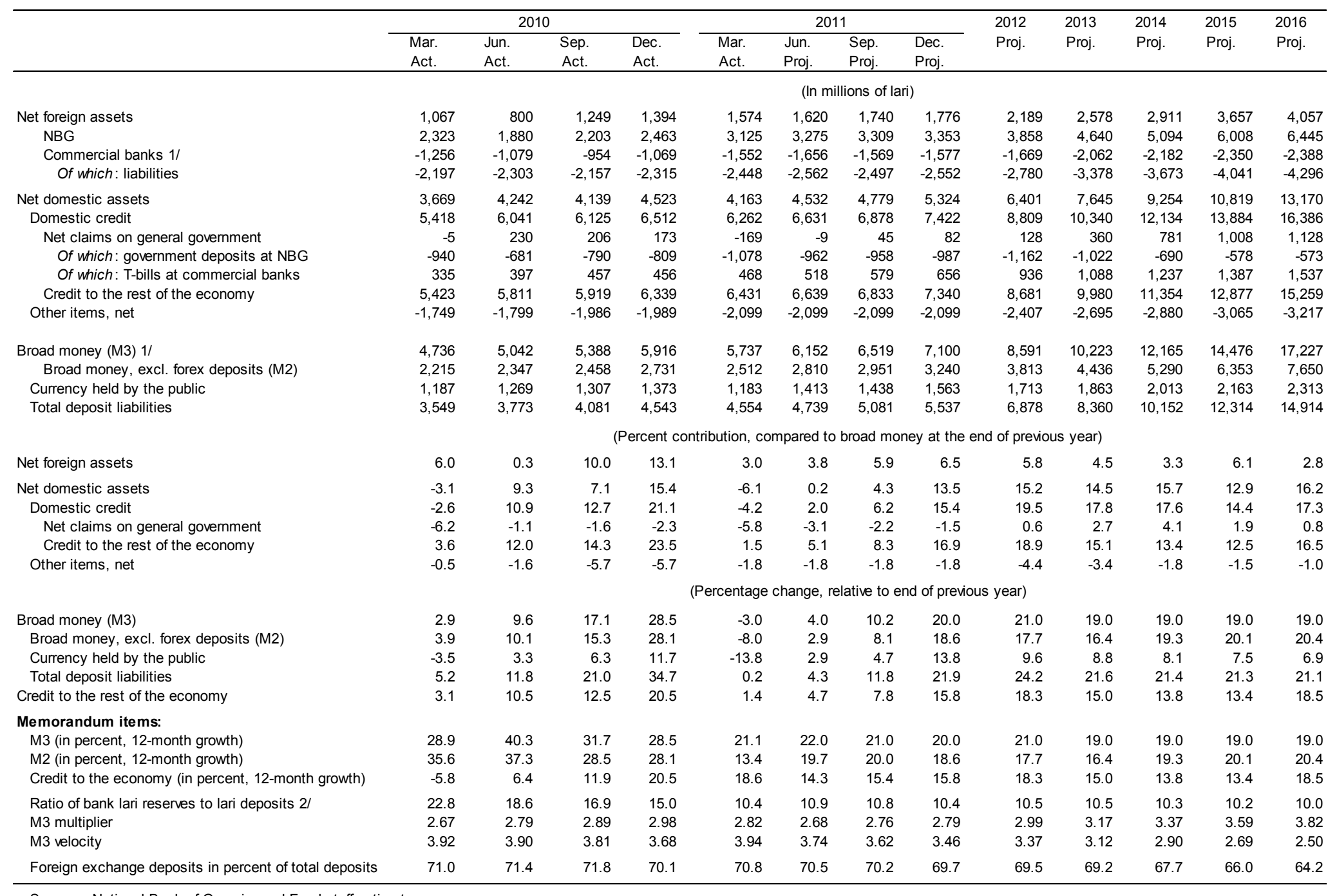

Sources: National Bank of Georgia; and Fund staff estimates.

1/ The proceeds of the Georgian Railway eurobond issuance from July 2010, which were deposited in accounts with Georgian commercial banks that placed them abroad, are subtracted from commercial banks' foreign assets and domestic fx deposits.

2/ Comprises required and excess reserves on lari-denominated deposits (excess reserves include overnight deposits with NBG). 
Table 6. Georgia: Selected Monetary and Financial Soundness Indicators, 2008-11

\begin{tabular}{|c|c|c|c|c|c|c|c|c|c|c|c|c|c|}
\hline & \multicolumn{4}{|c|}{2008} & \multicolumn{4}{|c|}{2009} & \multicolumn{4}{|c|}{2010} & \multirow{2}{*}{$\frac{2011}{\text { Mar }}$} \\
\hline & Mar. & Jun. & Sep. & $\overline{\text { Dec. }}$ & Mar. & Jun. & Sep. & $\overline{\text { Dec. }}$ & Mar. & Jun. & Sep. & $\overline{\text { Dec. }}$ & \\
\hline Deposit dollarization (in percent) & 60.9 & 60.0 & 61.7 & 75.7 & 75.2 & 73.2 & 72.6 & 73.2 & 71.0 & 71.4 & 71.8 & 70.1 & 70.8 \\
\hline Loan-to-deposit ratio (in percent) & 129.4 & 135.7 & 147.0 & 155.9 & 165.3 & 165.4 & 143.0 & 124.2 & 124.7 & 122.2 & 108.7 & 107.6 & 106.9 \\
\hline Credit-to-GDP ratio (in percent) & 28.5 & 30.0 & 29.2 & 31.9 & 30.5 & 30.0 & 29.3 & 29.2 & 29.6 & 30.5 & 29.8 & 30.5 & 29.9 \\
\hline Capital adequacy ratio (in percent) $1 /$ & 17.5 & 15.7 & 17.0 & 13.9 & 15.2 & 17.6 & 20.2 & 19.1 & 18.3 & 17.4 & 18.2 & 17.4 & 17.3 \\
\hline Capital adequacy ratio (in percent) $2 /$ & $\ldots$ & $\ldots$ & $\ldots$ & 24.0 & 23.6 & 27.1 & 27.4 & 25.6 & 24.6 & 23.3 & 24.4 & 23.6 & 27.5 \\
\hline Liquidity ratio (in percent) $3 /$ & 36.8 & 33.3 & 30.4 & 28.3 & 30.8 & 31.4 & 40.6 & 39.1 & 41.6 & 37.5 & 37.3 & 38.7 & 42.3 \\
\hline Nonperforming loans (in percent of total loans) $4 /$ & 3.0 & 3.4 & 9.9 & 12.8 & 15.2 & 18.8 & 18.2 & 17.9 & 17.1 & 16.3 & 15.1 & 12.5 & 11.9 \\
\hline Nonperforming loans (in percent of total loans) $5 /$ & $\ldots$ & $\ldots$ & $\ldots$ & 4.1 & 5.4 & 7.1 & 8.3 & 6.3 & 6.6 & 6.9 & 7.8 & 5.4 & 5.0 \\
\hline Loans collateralized by real estate (in percent of total loans) & 43.6 & 40.5 & 41.6 & 43.6 & 46.4 & 48.0 & 49.1 & 55.5 & 55.5 & 54.1 & 52.3 & 47.5 & 46.7 \\
\hline Loans in foreign exchange (in percent of total loans) & 65.9 & 64.9 & 67.5 & 72.8 & 75.3 & 77.3 & 77.6 & 76.9 & 75.9 & 73.7 & 72.8 & 74.0 & 73.4 \\
\hline Specific provisions (in percent of total loans) & 1.9 & 2.2 & 4.7 & 6.0 & 7.5 & 9.4 & 10.2 & 9.7 & 9.4 & 8.9 & 8.3 & 6.5 & 6.3 \\
\hline Net foreign assets (in percent of total assets) & -17.2 & -20.2 & -21.6 & -19.6 & -22.5 & -24.4 & -18.4 & -14.9 & -15.1 & -12.4 & -6.2 & -8.2 & -13.2 \\
\hline Net open foreign exchange position (in percent of regulatory capital) & 3.5 & 1.5 & 1.5 & 1.7 & 9.1 & 8.8 & 10.4 & 1.8 & 4.3 & 5.3 & 3.3 & 8.1 & 1.9 \\
\hline Return on equity (cumulative through the year, annualized) $6 /$ & 10.2 & 8.9 & -2.9 & -12.6 & -7.6 & -8.4 & -7.3 & -4.3 & 4.0 & 4.8 & 8.0 & 9.6 & 10.0 \\
\hline
\end{tabular}

Sources: National Bank of Georgia; and Fund staff estimates.

1/ National definition. Risk weight to forex loans was reduced from 200 to 175 percent in September 2008, and to 150 percent in August 2009 , and raised to 175 percent in January 2011.

2/ Basel I definition.

3/ Ratio of liquid assets to 6-month and shorter maturity liabilities.

4/ National definition: NPLs are defined as loans in substandard, doubtful, and loss loan categories.

5/ IMF definition.

6/ Pre tax. 
Table 7. Georgia: External Vulnerability Indicators, 2008-16

\begin{tabular}{|c|c|c|c|c|c|c|c|c|c|}
\hline & $\begin{array}{c}2008 \\
\text { Act. }\end{array}$ & $\begin{array}{c}2009 \\
\text { Act. }\end{array}$ & $\begin{array}{l}2010 \\
\text { Proj. }\end{array}$ & $\begin{array}{l}2011 \\
\text { Proj. }\end{array}$ & $\begin{array}{l}2012 \\
\text { Proj. }\end{array}$ & $\begin{array}{l}2013 \\
\text { Proj. }\end{array}$ & $\begin{array}{l}2014 \\
\text { Proj. }\end{array}$ & $\begin{array}{l}2015 \\
\text { Proj. }\end{array}$ & $\begin{array}{l}2016 \\
\text { Proj. }\end{array}$ \\
\hline Value of exports of goods and services, percent change & 15.9 & -13.0 & 26.6 & 24.2 & 10.0 & 6.0 & 4.7 & 6.2 & 7.0 \\
\hline Value of imports of goods and services, percent change & 26.8 & -29.8 & 15.7 & 21.5 & 5.2 & 1.4 & 2.5 & 4.1 & 7.2 \\
\hline Terms of trade (deterioration - ) & 0.4 & 1.3 & 3.4 & -0.3 & 0.9 & 0.1 & 0.5 & 1.1 & 1.4 \\
\hline Current account balance (percent of GDP) & -22.6 & -11.2 & -9.6 & -10.8 & -9.7 & -8.1 & -6.8 & -5.8 & -5.8 \\
\hline Capital and financial account (percent of GDP) & 22.5 & 15.0 & 8.6 & 15.1 & 10.5 & 10.6 & 8.8 & 8.0 & 6.4 \\
\hline External public debt (percent of GDP) & 20.9 & 31.4 & 33.7 & 31.4 & 30.4 & 30.1 & 29.1 & 28.7 & 28.3 \\
\hline in percent of exports of goods and services & 73.0 & 105.4 & 96.9 & 85.7 & 81.4 & 78.0 & 75.1 & 73.7 & 72.4 \\
\hline \multicolumn{10}{|l|}{ Debt service on external public debt } \\
\hline (in percent of exports of goods and services) & 3.4 & 5.2 & 4.1 & 13.1 & 7.5 & 11.0 & 7.7 & 5.1 & 4.3 \\
\hline External debt (percent of GDP) & 44.0 & 58.0 & 67.1 & 62.0 & 59.9 & 58.6 & 54.9 & 52.5 & 48.5 \\
\hline in percent of exports of goods and services & 153.6 & 194.9 & 192.7 & 169.0 & 160.3 & 151.8 & 141.7 & 134.9 & 124.2 \\
\hline \multicolumn{10}{|l|}{ Debt service on MLT external debt } \\
\hline (in percent of exports of goods and services) & 16.0 & 23.6 & 17.2 & 26.9 & 23.9 & 23.3 & 19.9 & 17.2 & 15.3 \\
\hline \multicolumn{10}{|l|}{ Gross international reserves } \\
\hline in millions of USD & 1,480 & 2,111 & 2,263 & 2,795 & 2,669 & 2,669 & 2,715 & 3,005 & 3,097 \\
\hline in months of next year's imports of goods and servces & 3.4 & 4.2 & 3.7 & 4.3 & 4.1 & 4.0 & 3.9 & 4.0 & 3.9 \\
\hline in percent of external debt & 26.1 & 33.8 & 28.9 & 32.8 & 30.0 & 29.9 & 31.1 & 34.1 & 35.7 \\
\hline in percent of short-term external debt (remaining maturity) & 101 & 165 & 111 & 133 & 129 & 141 & 151 & 168 & 165 \\
\hline
\end{tabular}

Source: Fund staff estimates and projections. 
Table 8. Georgia: Indicators of Fund Credit, 2008-17

(In millions of SDR)

\begin{tabular}{|c|c|c|c|c|c|c|c|c|c|c|}
\hline & 2008 & 2009 & 2010 & $\begin{array}{l}2011 \\
\text { Proj. }\end{array}$ & $\begin{array}{l}2012 \\
\text { Proj. }\end{array}$ & $\begin{array}{l}2013 \\
\text { Proj. }\end{array}$ & $\begin{array}{c}2014 \\
\text { Proj. }\end{array}$ & $\begin{array}{l}2015 \\
\text { Proj. }\end{array}$ & $\begin{array}{l}2016 \\
\text { Proj. }\end{array}$ & $\begin{array}{l}2017 \\
\text { Proj }\end{array}$ \\
\hline \multicolumn{11}{|l|}{ Existing Fund credit } \\
\hline Stock 1/ & 298.8 & 501.6 & 682.1 & 644.0 & 484.1 & 233.0 & 66.2 & 14.0 & 4.2 & 0.0 \\
\hline ECF 2/ & 137.1 & 119.1 & 105.0 & 87.1 & 67.2 & 47.6 & 29.4 & 14.0 & 4.2 & 0.0 \\
\hline SBA & 161.7 & 382.5 & 577.1 & 556.9 & 416.9 & 185.4 & 36.8 & 0.0 & 0.0 & 0.0 \\
\hline Obligations & 23.3 & 22.5 & 21.6 & 44.4 & 168.3 & 256.9 & 169.0 & 52.5 & 9.8 & 4.2 \\
\hline ECF 2/ & 22.8 & 18.6 & 14.1 & 17.9 & 20.1 & 19.7 & 18.3 & 15.5 & 9.8 & 4.2 \\
\hline SBA $3 /$ & 0.5 & 3.9 & 7.5 & 26.5 & 148.3 & 237.2 & 150.7 & 37.1 & 0.0 & 0.0 \\
\hline \multicolumn{11}{|l|}{ Prospective purchases under the SBA } \\
\hline Disbursements & $\ldots$ & $\ldots$ & 0.0 & 0.0 & 0.0 & 0.0 & 0.0 & 0.0 & 0.0 & 0.0 \\
\hline Stock 1/ & $\ldots$ & $\ldots$ & 0.0 & 0.0 & 0.0 & 0.0 & 0.0 & 0.0 & 0.0 & 0.0 \\
\hline Obligations $3 /$ & $\ldots$ & $\ldots$ & 0.0 & 0.0 & 0.0 & 0.0 & 0.0 & 0.0 & 0.0 & 0.0 \\
\hline Principal (repurchases) & $\ldots$ & $\ldots$ & 0.0 & 0.0 & 0.0 & 0.0 & 0.0 & 0.0 & 0.0 & 0.0 \\
\hline GRA charges & $\ldots$ & $\ldots$ & 0.0 & 0.0 & 0.0 & 0.0 & 0.0 & 0.0 & 0.0 & 0.0 \\
\hline Surcharges & $\ldots$ & $\ldots$ & 1.4 & 1.9 & 1.6 & 0.0 & 0.0 & 0.0 & 0.0 & 0.0 \\
\hline Stock of existing and prospective Fund credit $1 /$ & 298.8 & 501.6 & 682.1 & 644.0 & 484.1 & 233.0 & 66.2 & 14.0 & 4.2 & 0.0 \\
\hline In percent of quota & 198.8 & 333.7 & 453.8 & 428.5 & 322.1 & 155.0 & 44.1 & 9.3 & 2.8 & 0.0 \\
\hline In percent of GDP & 3.7 & 7.2 & 8.9 & 7.3 & 5.1 & 2.4 & 0.7 & 0.1 & 0.0 & 0.0 \\
\hline In percent of exports of goods and nonfactor services & 12.8 & 24.1 & 25.6 & 20.0 & 13.6 & 6.2 & 1.7 & 0.3 & 0.1 & 0.0 \\
\hline In percent of gross reserves & 31.9 & 36.7 & 46.0 & 36.1 & 28.3 & 13.6 & 3.8 & 0.7 & 0.2 & 0.0 \\
\hline In percent of public external debt & 17.5 & 22.9 & 26.4 & 23.3 & 16.8 & 7.9 & 2.2 & 0.5 & 0.1 & 0.0 \\
\hline \multicolumn{11}{|l|}{ Obligations to the Fund from existing and } \\
\hline In percent of quota & 15.5 & 15.0 & 15.3 & 30.8 & 113.1 & 170.9 & 112.4 & 35.0 & 6.5 & 2.8 \\
\hline In percent of GDP & 0.3 & 0.3 & 0.3 & 0.5 & 1.8 & 2.6 & 1.7 & 0.5 & 0.1 & 0.0 \\
\hline In percent of exports of goods and nonfactor services & 1.0 & 1.1 & 0.9 & 1.4 & 4.8 & 6.8 & 4.3 & 1.3 & 0.2 & 0.1 \\
\hline In percent of gross reserves & 2.5 & 1.6 & 1.5 & 2.6 & 10.0 & 15.0 & 9.7 & 2.7 & 0.5 & 0.2 \\
\hline In percent of public external debt service & 29.3 & 20.7 & 20.9 & 10.9 & 64.1 & 61.9 & 55.3 & 24.4 & 5.0 & 1.8 \\
\hline
\end{tabular}

Source: Fund staff estimates and projections.

$1 /$ End of period.

2/ Following the Low Income Countries (LIC) reforms, effective January 7 2010, the PRGF arrangements were renamed Extended Credit Facility (ECF) arrangements.

3/ Repayment schedule based on repurchase obligations and GRA charges. 
Table 9. Georgia: Schedule of Prospective Reviews and Purchases 1/

15-Sep-08 Approve the 18-month arrangement

15-Dec-08 Complete the first review based on end-September 2008 performance criteria and other relevant performance criteria 2/

23-Mar-09 Complete the second review based on end-December 2008 performance criteria and other relevant performance criteria $2 /$

6-Aug-09 Complete the third review based on end-June 2009 performance criteria and other relevant performance criteria

15-Nov-09 Complete the fourth review based on end-September 2009 performance criteria and other relevant performance criteria

15-Feb-10 Complete the fifth review based on end-December 2009 performance criteria and other relevant performance criteria

9-Jul-10 Complete the sixth review based on end-June 2010 performance criteria and other relevant performance criteria

15-Nov-10 Complete the seventh and eighth reviews based on end-December 2010 performance criteria and other relevant performance criteria

15-May-11 Complete the ninth review based on end-March 2011 performance criteria and other relevant performance criteria

Total available
161.7

1/ Reflects the augmentation of access by SDR270 million and the extended arrangement through June 2011.

2/ As the authorities did not draw the purchase that became available at the time of the first review, SDR 126.2 million were available and purchased at the second review. 
Table 10. Georgia: External Financing Requirements and Sources, 2008-16 (In millions of U.S. dollars)

\begin{tabular}{|c|c|c|c|c|c|c|c|c|c|}
\hline & 2008 & 2009 & 2010 & 2011 & 2012 & 2013 & 2014 & 2015 & 2016 \\
\hline Total requirements & $-3,275$ & $-1,721$ & $-1,550$ & $-2,491$ & $-2,371$ & $-2,169$ & $-1,892$ & $-1,677$ & $-1,727$ \\
\hline Current account deficit & $-2,912$ & $-1,210$ & $-1,117$ & $-1,481$ & $-1,439$ & $-1,228$ & $-1,085$ & -970 & $-1,039$ \\
\hline Capital outflows: Repayments of MLT loans & -363 & -511 & -434 & $-1,010$ & -931 & -941 & -808 & -707 & -688 \\
\hline Total sources & 3,275 & 1,721 & 1,550 & 2,491 & 2,371 & 2,169 & 1,892 & 1,677 & 1,727 \\
\hline Capital flows & 3,188 & 2,145 & 1,498 & 3,022 & 2,244 & 2,169 & 1,938 & 1,967 & 1,819 \\
\hline Public sector & 795 & 872 & 592 & 1,007 & 505 & 593 & 376 & 378 & 379 \\
\hline Project grants & 87 & 170 & 189 & 117 & 60 & 50 & 40 & 39 & 38 \\
\hline Long-term loan disbursements to public sector & 227 & 428 & 401 & 421 & 345 & 343 & 336 & 339 & 341 \\
\hline Other & 482 & 274 & 1 & 469 & 100 & 200 & 0 & 0 & 0 \\
\hline Of which: unidentified & 0 & 0 & 0 & 0 & 100 & 200 & 0 & 0 & 0 \\
\hline Private sector & 2,392 & 1,273 & 906 & 2,015 & 1,739 & 1,576 & 1,562 & 1,589 & 1,441 \\
\hline Foreign direct investment in Georgia & 1,564 & 658 & 499 & 712 & 806 & 865 & 877 & 928 & 989 \\
\hline Long-term loan disbursements to private sector & 759 & 575 & 301 & 781 & 795 & 711 & 571 & 579 & 584 \\
\hline Other net inflows $1 /$ & 69 & 40 & 107 & 523 & 139 & -1 & 113 & 82 & -132 \\
\hline Financing & 218 & 192 & 260 & 0 & 0 & 0 & 0 & 0 & 0 \\
\hline IMF 2/ & 257 & 340 & 297 & 0 & 0 & 0 & 0 & 0 & 0 \\
\hline Change in arrears, net (- decrease) & -9 & 27 & 15 & 0 & 0 & 0 & 0 & 0 & 0 \\
\hline Advance Repayments & -29 & -186 & -63 & 0 & 0 & 0 & 0 & 0 & 0 \\
\hline Change in reserves (- increase) & -131 & -616 & -208 & -531 & 126 & 0 & -46 & -290 & -93 \\
\hline \multicolumn{10}{|l|}{ Memorandum items (in percent of GDP): } \\
\hline Total financing requirements & -25.4 & -16.0 & -13.3 & -18.1 & -16.0 & -14.2 & -11.9 & -10.0 & -9.7 \\
\hline Total sources & 25.4 & 16.0 & 13.3 & 18.1 & 16.0 & 14.2 & 11.9 & 10.0 & 9.7 \\
\hline Capital inflows & 24.8 & 19.9 & 12.8 & 22.0 & 15.1 & 14.2 & 12.2 & 11.7 & 10.2 \\
\hline Exceptional financing & 1.7 & 1.8 & 2.2 & 0.0 & 0.0 & 0.0 & 0.0 & 0.0 & 0.0 \\
\hline Change in reserves (- increase) & -1.0 & -5.7 & -1.8 & -3.9 & 0.9 & 0.0 & -0.3 & -1.7 & -0.5 \\
\hline
\end{tabular}

Sources: Georgian Statistics Department; National Bank of Georgia; and Fund staff estimates.

$1 /$ Including errors and omissions.

2/ SBA, including augmentation in 2010-11. 
Table 11. Georgia: External Debt Sustainability Framework, 2006-2016

(In percent of GDP, unless otherwise indicated)

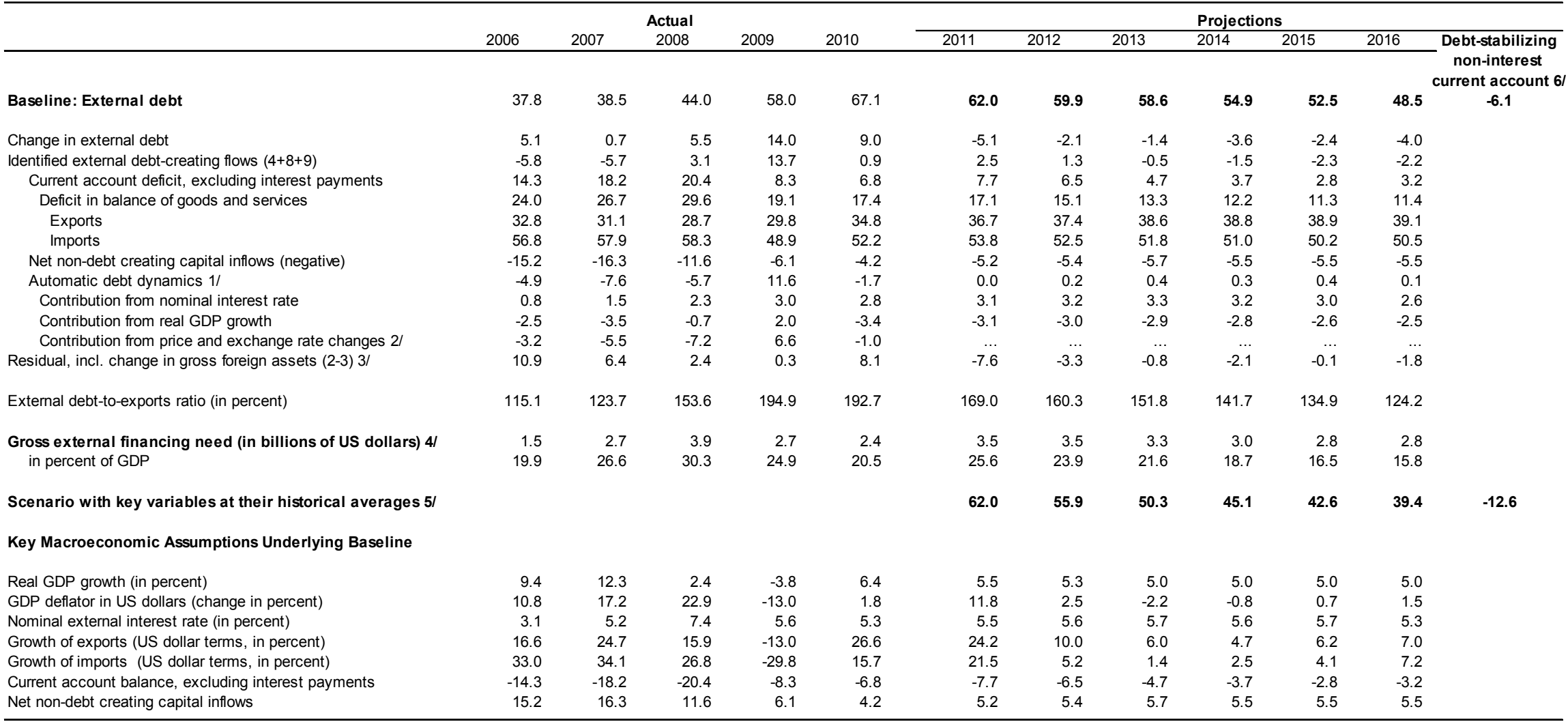

$1 /$ Derived as $[r-g-\rho(1+g)+s \alpha(1+r)](1+g+\rho+g \rho)$ times previous period debt stock, with $r=$ nominal effective interest rate on external debt; $\rho=$ change in domestic GDP deflator in US dollar terms, $g=$ real GDP growth rate

$\varepsilon=$ nominal appreciation (increase in dollar value of domestic currency), and $\alpha=$ share of domestic-currency denominated debt in total external debt.

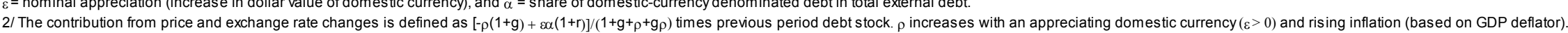

$3 /$ For projection, line includes the impact of price and exchange rate changes.

4/ Defined as current account deficit, plus amortization on medium- and long-term debt, plus short-term debt at end of previous period.

5/ The key variables include real GDP growth; nominal interest rate; dollar deflator growth; and both non-interest current account and non-debt inflows in percent of GDP.

6/ Long-run, constant balance that stabilizes the debt ratio assuming that key variables (real GDP growth, nominal interest rate, dollar deflator growth, and non-debt inflows in percent of GDP) remain at their levels

of the last projection year. 
Table 12. Georgia: Public Sector Debt Sustainability Framework, 2006-2016 (In percent of GDP, unless otherwise indicated)

\begin{tabular}{|c|c|c|c|c|c|c|c|c|c|c|c|c|}
\hline & \multicolumn{5}{|c|}{ Actual } & \multicolumn{6}{|c|}{ Projections } & \multirow{3}{*}{$\begin{array}{c}\text { Debt-stabilizing } \\
\text { primary } \\
\text { balance } 9 /\end{array}$} \\
\hline & 2006 & 2007 & 2008 & 2009 & 2010 & 2011 & 2012 & 2013 & 2014 & 2015 & 2016 & \\
\hline & & & & & & & & & & & & \\
\hline \multirow{2}{*}{$\begin{array}{l}\text { Baseline: Public sector debt } 1 / \\
\text { o/w foreign-currency denominated }\end{array}$} & 27.3 & 21.5 & 27.6 & 37.3 & 39.1 & 36.8 & 37.6 & 37.0 & 35.5 & 34.8 & 34.0 & -1.1 \\
\hline & 21.3 & 16.8 & 23.5 & 31.7 & 33.6 & 31.3 & 31.7 & 31.2 & 29.8 & 29.3 & 28.7 & \\
\hline Change in public sector debt & -6.8 & -5.8 & 6.1 & 9.7 & 1.8 & -2.2 & 0.8 & -0.6 & -1.5 & -0.7 & -0.8 & \\
\hline Identified debt-creating flows $(4+7+12)$ & -8.3 & -7.0 & 1.0 & 9.1 & 1.9 & -1.9 & -1.1 & -1.0 & -1.7 & -1.7 & -1.8 & \\
\hline Primary deficit & 2.3 & 4.2 & 5.7 & 8.2 & 5.6 & 2.2 & 2.1 & 1.6 & 0.8 & 0.5 & 0.3 & \\
\hline Revenue and grants & 26.7 & 29.3 & 30.7 & 29.3 & 28.2 & 28.5 & 26.5 & 26.3 & 26.1 & 26.1 & 26.1 & \\
\hline Primary (noninterest) expenditure & 29.0 & 33.4 & 36.4 & 37.5 & 33.8 & 30.7 & 28.6 & 27.9 & 26.9 & 26.6 & 26.4 & \\
\hline Automatic debt dynamics $2 /$ & -5.4 & -6.0 & -1.0 & 2.9 & -2.6 & -3.2 & -2.8 & -2.5 & -2.3 & -2.2 & -2.1 & \\
\hline Contribution from interest rate/growth differential $3 /$ & -4.6 & -4.6 & -1.7 & 2.6 & -4.0 & -3.2 & -2.8 & -2.5 & -2.3 & -2.2 & -2.1 & \\
\hline Of which contribution from real interest rate & -1.9 & -1.8 & -1.3 & 1.5 & -2.0 & -1.4 & -1.1 & -0.8 & -0.6 & -0.6 & -0.6 & \\
\hline Of which contribution from real GDP growth & -2.7 & -2.7 & -0.5 & 1.1 & -2.1 & -1.9 & -1.7 & -1.7 & -1.7 & -1.6 & -1.6 & \\
\hline Contribution from exchange rate depreciation 4/ & -0.8 & -1.4 & 0.7 & 0.3 & 1.4 & $\ldots$ & $\ldots$ & $\ldots$ & $\ldots$ & $\ldots$ & $\ldots$ & \\
\hline Other identified debt-creating flows & -5.2 & -5.2 & -3.7 & -2.0 & -1.1 & -0.8 & -0.4 & -0.2 & -0.2 & 0.0 & 0.0 & \\
\hline Privatization receipts (negative) & -5.2 & -5.2 & -3.7 & -2.0 & -1.1 & -0.8 & -0.4 & -0.2 & -0.2 & 0.0 & 0.0 & \\
\hline Recognition of implicit or contingent liabilities & 0.0 & 0.0 & 0.0 & 0.0 & 0.0 & 0.0 & 0.0 & 0.0 & 0.0 & 0.0 & 0.0 & \\
\hline Other (specify, e.g. bank recapitalization) & 0.0 & 0.0 & 0.0 & 0.0 & 0.0 & 0.0 & 0.0 & 0.0 & 0.0 & 0.0 & 0.0 & \\
\hline Residual, including asset changes (2-3) 5/ & 1.5 & 1.3 & 5.1 & 0.5 & -0.2 & -0.4 & 1.9 & 0.4 & 0.2 & 1.0 & 1.0 & \\
\hline Public sector debt-to-revenue ratio $1 /$ & 102.5 & 73.6 & 90.0 & 127.4 & 138.5 & 129.4 & 142.2 & 140.7 & 135.9 & 133.3 & 130.5 & \\
\hline Gross financing need 6 / & 5.2 & 5.7 & 6.9 & 10.1 & 8.6 & 9.2 & 6.2 & 8.0 & 7.2 & 6.3 & 5.9 & \\
\hline in billions of U.S. dollars & 0.4 & 0.6 & 0.9 & 1.1 & 1.0 & 1.3 & 0.9 & 1.2 & 1.2 & 1.1 & 1.1 & \\
\hline Scenario with key variables at their historical averages $7 /$ & & & & & & 36.8 & 37.5 & 36.9 & 36.1 & 36.4 & 36.7 & -2.5 \\
\hline Scenario with no policy change (constant primary balance) in 2011-2016 & & & & & & 36.8 & 37.7 & 37.7 & 37.6 & 38.6 & 39.6 & -1.3 \\
\hline \multicolumn{13}{|l|}{ Key Macroeconomic and Fiscal Assumptions Underlying Baseline } \\
\hline Real GDP growth (in percent) & 9.4 & 12.3 & 2.4 & -3.8 & 6.4 & 5.5 & 5.3 & 5.0 & 5.0 & 5.0 & 5.0 & \\
\hline Average nominal interest rate on public debt (in percent) $8 /$ & 2.6 & 2.6 & 3.3 & 3.2 & 3.1 & 4.0 & 3.5 & 3.7 & 3.7 & 3.8 & 3.8 & \\
\hline Average real interest rate (nominal rate minus change in GDP deflator, in percer & -5.9 & -7.1 & -6.3 & 5.2 & -5.6 & -3.5 & -3.0 & -2.0 & -1.6 & -1.5 & -1.5 & \\
\hline Nominal appreciation (increase in US dollar value of local currency, in percent) & 3.6 & 8.7 & -4.5 & -1.1 & -4.9 & & & & & & $\cdots$ & \\
\hline Inflation rate (GDP deflator, in percent) & 8.5 & 9.7 & 9.6 & -2.0 & 8.7 & 7.5 & 6.5 & 5.7 & 5.3 & 5.3 & 5.2 & \\
\hline Growth of real primary spending (deflated by GDP deflator, in percent) & 26.7 & 29.7 & 11.5 & -0.9 & -4.1 & -4.2 & -1.9 & 2.4 & 1.1 & 3.8 & 4.3 & \\
\hline Primary deficit & 2.3 & 4.2 & 5.7 & 8.2 & 5.6 & 2.2 & 2.1 & 1.6 & 0.8 & 0.5 & 0.3 & \\
\hline
\end{tabular}

$1 /$ Indicate coverage of public sector, e.g., general government or nonfinancial public sector. Also whether net or gross debt is used.

$2 /$ Derived as $[(r-\pi(1+g)-g+\alpha \varepsilon(1+r)](1+g+\pi+g \pi))$ times previous period debt ratio, with $r=$ interest rate; $\pi=$ growth rate of GDP deflator; $g=$ real GDP growth rate; $\alpha=$ share of foreign-currency denominated debt; and $\varepsilon=$ nominal exchange rate depreciation (measured by increase in local currency value of U.S. dollar).

$3 /$ The real interest rate contribution is derived from the denominator in footnote $2 /$ as $r-\pi(1+g)$ and the real growth contribution as $-g$.

$4 /$ The exchange rate contribution is derived from the numerator in footnote $2 /$ as $\alpha \varepsilon(1+r)$.

$5 /$ For projections, this line includes exchange rate changes.

6/ Defined as public sector deficit, plus amortization of medium and long-term public sector debt, plus short-term debt at end of previous period.

7/ The key variables include real GDP growth; real interest rate; and primary balance in percent of GDP.

$8 /$ Derived as nominal interest expenditure divided by previous period debt stock.

9/ Assumes that key variables (real GDP growth, real interest rate, and other identified debt-creating flows) remain at the level of the last projection year.

\section{OInternational Monetary Fund. Not for Redistribution}


Figure 1. Georgia: External Debt Sustainability: Bound Tests 1/ (External debt in percent of GDP)
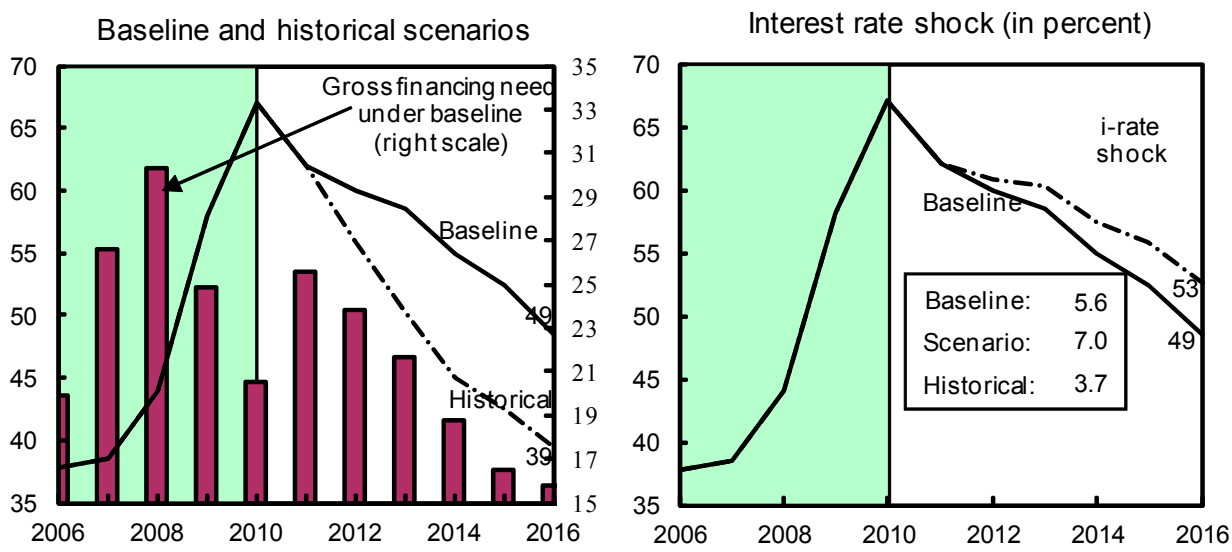

Growth shock (in percent per year)

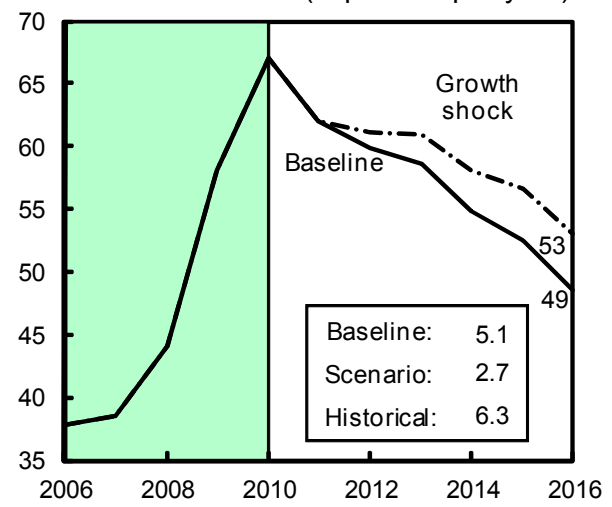

Non-interest current account shock (in percent of GDP)

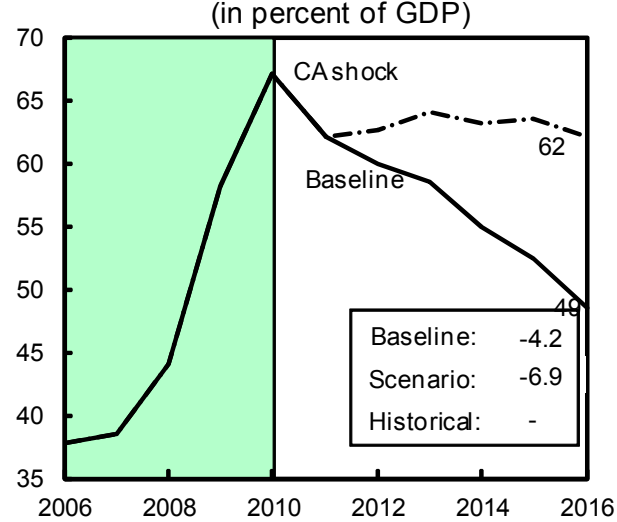

Combined shock 2/
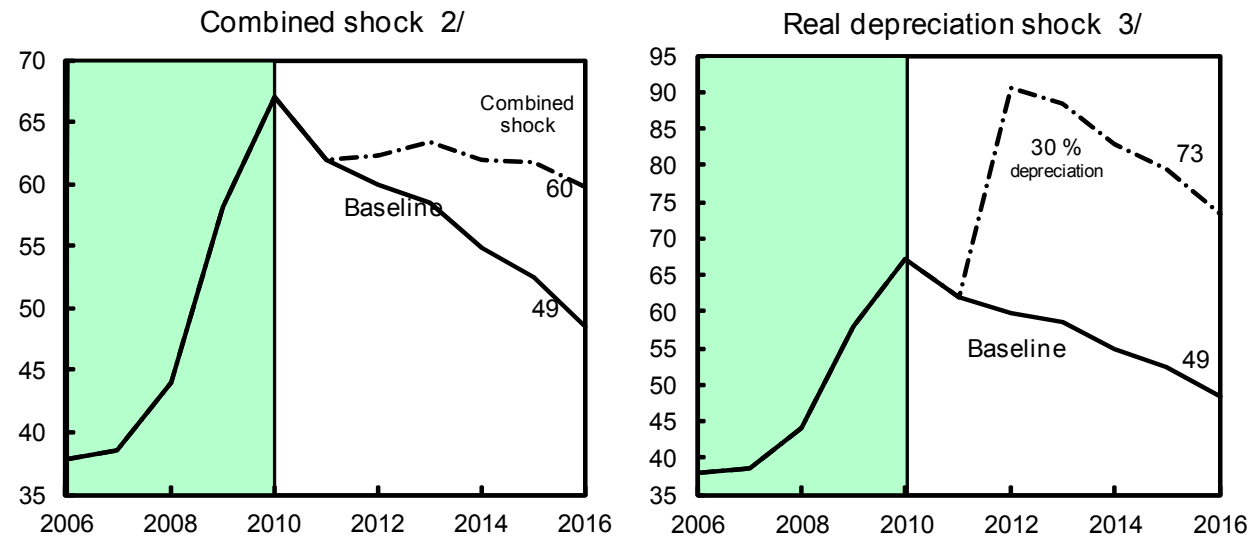

Sources: International Monetary Fund, Country desk data, and staff estimates.

$1 /$ Shaded areas represent actual data. Individual shocks are permanent one-half standard deviation shocks. Figures in the boxes represent average projections for the respective variables in the baseline and scenario being presented. Ten-year historical average for the variable is also shown. $2 /$ Permanent $1 / 4$ standard deviation shocks applied to real interest rate, growth rate, and current account balance.

3/One-time real depreciation of 30 percent occurs in 2010 . 
Figure 2. Georgia: Public Debt Sustainability: Bound Tests 1/ (Public debt in percent of GDP)

Baseline and historical scenarios

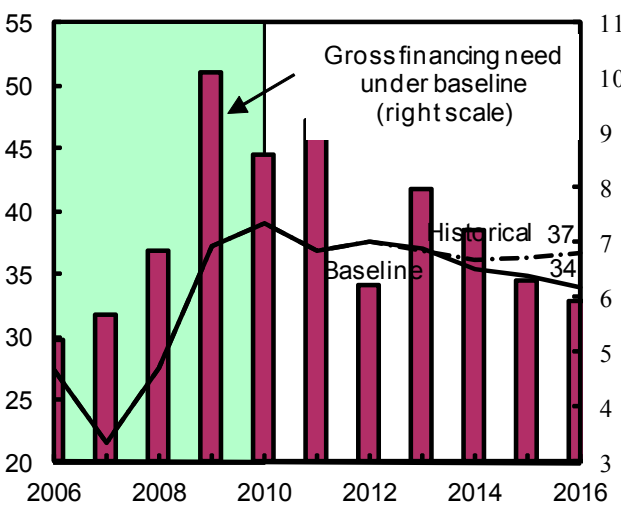

Growth shock (in percent per

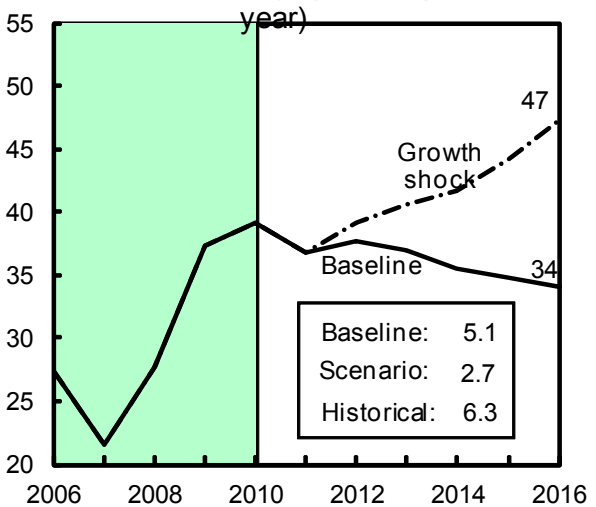

Combined shock 2/

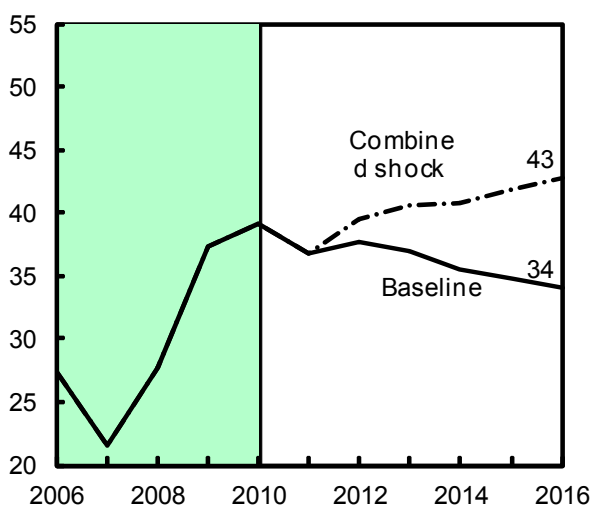

Interest rate shock (in percent)

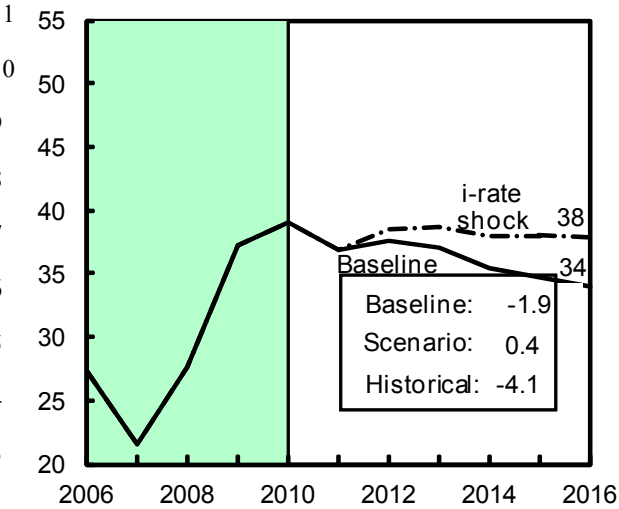

Primary balance shock (in percent of GDP) and no policy change scenario (constant primary

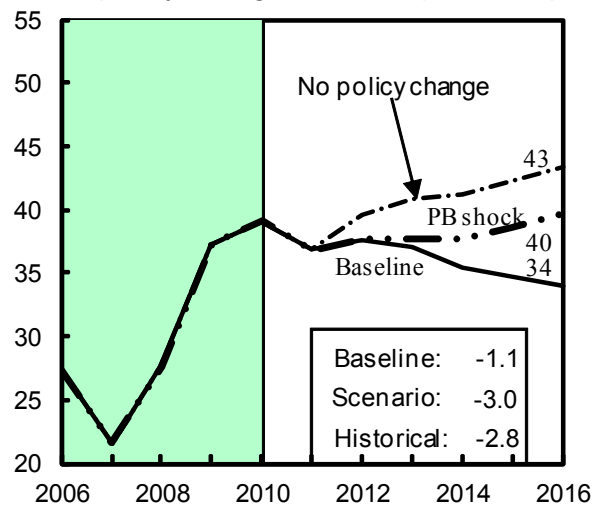

Real depreciation and contingent liabilities shocks 3/

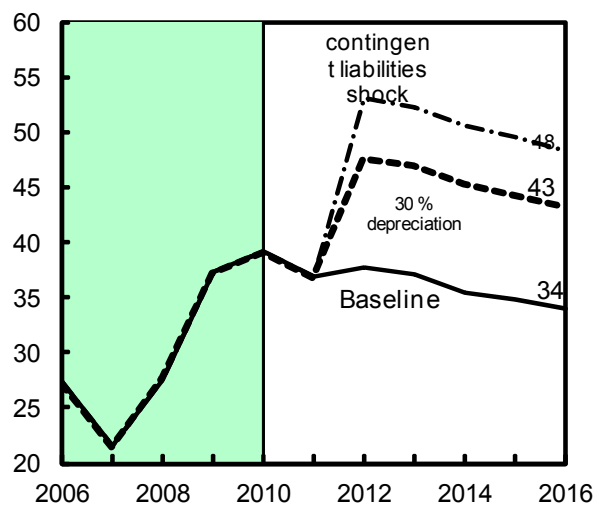

Sources: International Monetary Fund, country desk data, and staff estimates.

1/ Shaded areas represent actual data. In dividual shocks are permanent one-half standard deviation shocks.

Figures in the boxes represent average projections for the respective variables in the baseline and scenario being presented. Ten-year historical average for the variable is also shown.

2/ Permanent 1/4 standard deviation shocks ap plied to real interest rate, growth rate, and primary balance.

$3 /$ One-time real depreciation of 30 percent and 10 percent of GDP shock to contingent liabilities occur in 2010 , with real depreciation defined as nominal depreciation (measured by percentage fall in dollar value of local curren cy) minus domestic inflation (based on GDP deflator). 


\section{ATTACHMENT I. GEORgIA: LETTER OF INTENT}

May 24, 2011

The Acting Managing Director International Monetary Fund Washington, D.C.

Dear Mr. Lipsky:

1. In September 2008, the International Monetary Fund (IMF) approved a Stand-By Arrangement (SBA) for Georgia, which was further augmented in August 2009. On January 12, 2011, the IMF's Executive Board completed the seventh and eighth reviews under the SBA. We are grateful for the assistance which the IMF has been providing to Georgia.

2. This letter of intent describes the economic policies that we plan to implement during the remainder of 2011, consistent with our commitment to maintain macroeconomic stability and facilitate the process of macroeconomic adjustment.

3. Policies beyond 2011 will be guided by the same objectives of attaining sound fiscal and balance of payments positions, and maintaining low inflation. We are presently elaborating a comprehensive economic plan to reach these objectives, and we will request Fund support for this plan through a successor arrangement in the near future.

\section{Recent Economic Developments}

4. Recent economic developments have been broadly in line with program projections:

- Positive developments for the first quarter economic indicators are consistent with an annual real GDP growth projection of 5.5 percent in 2011.

- $\quad$ CPI inflation peaked at 13.9 percent in March under the impetus of rising food and fuel prices, and has since begun to come down. Core inflation (defined as the CPI excluding food, beverages, tobacco, and energy) remains subdued, and has recently declined below 2 percent, pointing to the absence of second-round price effects. Based on an expected stabilization of commodity prices, we project CPI inflation to decelerate in the second half of the year to around 8.5 percent by year-end.

- Balance-of-payment developments in the first quarter of the year were more favorable than anticipated. Despite higher import prices, which resulted in a widening of the trade deficit in the first quarter by almost 50 percent year-on-year, other inflows 
turned out stronger than projected, notably tourism receipts, which grew by over 40 percent year-on-year. Also, net financial inflows to the private sector are estimated to have risen sharply, as banks had to meet higher reserve requirements and reflecting inflows to meet the spending needs of key corporate clients, notably the railway company.

- $\quad$ On April 12, Georgia successfully issued a \$500-million Eurobond at very favorable terms (10-year maturity and 7.125-percent yield), reducing significantly medium-term debt rollover risks and establishing an excellent sovereign benchmark in the international capital markets. The proceeds of the issue were used to buy back most of the $\$ 500$ million Eurobond maturing in 2013.

- Better-than-expected external developments contributed to an appreciation of the lari and also enabled the central bank to accumulate net international reserves in excess of program targets. Since the beginning of the year the lari has appreciated by 5.7 percent against the dollar, and by 0.1 percent against the euro. Over the same period, the central bank has purchased $\$ 20$ million, on a net basis, on the foreign exchange market. As a result, net international reserves have reached their pre-crisis level.

- $\quad$ The growth rates of monetary and credit aggregates are currently at levels consistent with projected GDP growth and inflation. Broad money increased by 21 percent yearon year in March (down from 40 percent in mid-2010), while credit grew by 19 percent.

- $\quad$ Banking sector balance sheets remain sound. NPLs have declined further to 5 percent of the loan portfolio in March 2011 (by the IMF definition). Capitalization, provisioning, and liquidity ratios are within comfortable margins, bank profitability has increased, and reliance on external wholesale funding has declined significantly relative to the pre-crisis level.

\section{Policies for 2011}

5. Notwithstanding the achievements made under the program, additional efforts will be needed to ensure macroeconomic stability over the medium term. To that end, we intend to maintain the broad policy objectives to which we committed at the time of the seventh and eighth reviews. 
6. The recently adopted supplementary budget for 2011 targets a larger reduction in the deficit that envisaged at the time of the seventh and eighth reviews, as higher tax revenue would largely be saved and would be only partly used to finance additional social and capital outlays. Social spending was increased by the one-off distribution of food and electricity vouchers and a 25 percent increase in basic pensions scheduled for September. The budgeted increase in capital spending reflects infrastructure upgrades across a number of sectors. Overall, we stand ready to keep spending below budgeted levels in order to achieve the desired adjustment, given the uncertainty about the recurrent component of the revenue gain. On that basis, we are committed to reducing the government fiscal deficit by 3 percentage points of GDP, from 6.6 percent in 2010 to 3.6 percent in 2011.

7. We remain committed to a flexible exchange rate. Intervention, in either direction, will be motivated by the need to dampen excess volatility in the very thin Georgian foreign exchange market. At the same time, based on the results of the first quarter, we have also revised up our annual target for net international reserves (NIR), which we now project to reach $\$ 1.1$ billion by year-end.

8. We consider that the monetary policy stance is consistent with the projected deceleration of inflation. We will continue to monitor closely developments in headline and core inflation and credit growth, and stand ready to adjust our monetary policy stance in the event these indicators deviate from current projections.

\section{Completion of the Review}

9. All but one of the end-March 2011 performance criteria (PCs) were met. The endMarch PC on general government expenditure was missed owing to the distribution of food and electricity vouchers (aimed at alleviating the social impact of the hike in food and fuel prices) and an accelerated pace of project implementation under the Millennium Challenge Georgia Fund. At the same time, other current spending was lower than expected. We consider that the nonobservance of this $\mathrm{PC}$ does not compromise the program objectives and that it is more than offset by our revised (and tighter) deficit target for the year.

10. Based on our performance under the program and the explanations provided in this letter, we request a waiver of nonobservance of the end-March PC on general government expenditures and completion of the ninth review. We will maintain our usual close policy dialogue with the Fund. 
11. We authorize the IMF to publish this Letter of Intent as well as the accompanying staff report.

Sincerely yours,

/s/

Nika Gilauri

Prime Minister of Georgia /s/

Kakha Baindurashvili Minister of Finance

/s/

Giorgi Kadagidze

President of the National Bank of Georgia 
Table 1. Georgia: Quantitative Performance Criteria (PC) and Indicative Targets, 2010-11

\begin{tabular}{|c|c|c|c|c|c|c|}
\hline \multirow[b]{2}{*}{ Performance Criteria } & \multicolumn{3}{|c|}{ Dec-10 } & \multicolumn{3}{|c|}{ Mar-11 } \\
\hline & $\mathrm{PC}$ & Adjusted PC & Actual & $\mathrm{PC}$ & Adjusted PC & Actual \\
\hline & \multicolumn{6}{|c|}{ (Cumulative change since the beginning of the year, in millions of lari) } \\
\hline Ceiling on cash deficit of the general government & 1,241 & $\ldots$ & 1,366 & 170 & 138 & -197 \\
\hline \multirow[t]{2}{*}{ Ceiling on the general government expenditures } & 7,106 & $\ldots$ & 7,232 & 1,553 & 1,521 & 1,568 \\
\hline & \multicolumn{6}{|c|}{ (End-period stock, in millions of lari) } \\
\hline \multirow[t]{2}{*}{ Ceiling on net domestic assets (NDA) of the NBG 1/ } & 714 & 689 & 349 & 516 & 543 & -126 \\
\hline & \multicolumn{6}{|c|}{ (End-period stock, in millions of U.S. dollars) } \\
\hline \multirow[t]{2}{*}{ Floor on net international reserves (NIR) of the NBG 1/ } & 721 & 736 & 882 & 757 & 741 & 994 \\
\hline & \multicolumn{6}{|c|}{ (Cumulative change since the beginning of the year, in millions of U.S. dollars) } \\
\hline Ceiling on accumulation of external arrears $2 /$ & 0 & $\cdots$ & 0 & 0 & $\ldots$ & 0 \\
\hline \multirow[t]{2}{*}{ Indicative target } & Ind. Target & Adjusted Target & Actual & Ind. Target & Adjusted Target & Proj. \\
\hline & \multicolumn{6}{|c|}{ (Cumulative change since the beginning of the year, in millions of U.S. dollars) } \\
\hline $\begin{array}{l}\text { Ceiling on contracting or guaranteeing of new total external debt } \\
\text { by the public sector }\end{array}$ & 1,000 & $\ldots$ & 718 & 500 & 500 & 209 \\
\hline
\end{tabular}

Sources: Georgian authorities; and Fund staff estimates.

1/ Actual figures and quantitative targets are based on program exchange rates.

$2 /$ The continuous performance criterion for external arrears is defined in paragraph 21 of the TMU (Country Report No. 11/31). 


\section{GEORGIA}

Ninth Review Under the Stand-By Arrangement and Request for Waiver of Nonobservance of Performance Criterion

\section{Informational Annex}

Prepared by the Middle East and Central Asia Department

May 24, 2011

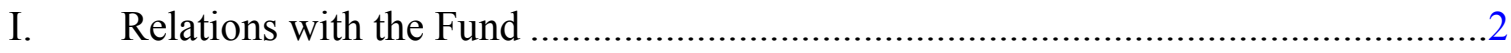

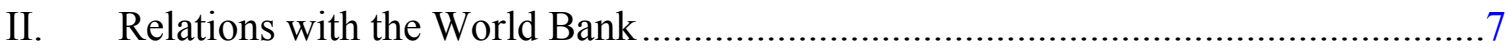

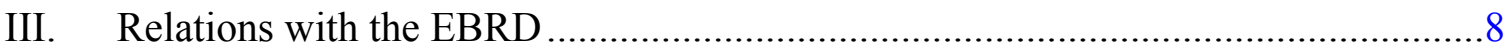




\section{ANNEX I. GEORGIA: RELATIONS WITH THE FUND}

(As of March 31, 2011)

I. Membership Status: Georgia joined the Fund on May 5, 1992.

II. General Resources Account:

Quota

Fund holdings of currency

Reserve position in Fund

III. SDR Department:

Holdings

IV. Outstanding Purchases and Loans:

Stand-by Arrangements

$\mathrm{ECF}^{1}$
SDR Million

150.30

727.40

0.01

SDR Million

147.56

SDR Million

577.10

98.15

\section{Percent of Quota}

100.00

483.97

0.01

Percent of Allocation

102.50

Percent of Quota

383.97

65.30

V. Latest Financial Arrangements:

\begin{tabular}{|c|c|c|c|c|}
\hline Type & $\begin{array}{l}\text { Approval } \\
\text { Date }\end{array}$ & $\begin{array}{c}\text { Expiration } \\
\text { Date }\end{array}$ & $\begin{array}{l}\text { Amount Approved } \\
\text { (SDR million) }\end{array}$ & $\begin{array}{l}\text { Amount Drawn } \\
\text { (SDR Million) }\end{array}$ \\
\hline Stand-By & $9 / 15 / 08$ & $6 / 14 / 11$ & 747.10 & 577.10 \\
\hline $\mathrm{ECF}^{1}$ & $6 / 4 / 04$ & $9 / 30 / 07$ & 98.00 & 98.00 \\
\hline $\mathrm{ECF}^{1}$ & $1 / 12 / 01$ & $1 / 11 / 04$ & 108.00 & 49.50 \\
\hline
\end{tabular}

VI. Projected Payments to Fund (Expectation Basis):

(SDR million; based on existing use of resources and present holdings of SDRs):

\begin{tabular}{crrrrr} 
& \multicolumn{5}{c}{ Forthcoming } \\
\cline { 2 - 6 } & $\mathbf{2 0 1 1}$ & $\mathbf{2 0 1 2}$ & $\mathbf{2 0 1 3}$ & $\mathbf{2 0 1 4}$ & $\mathbf{2 0 1 5}$ \\
Principal & 31.26 & 159.90 & 251.11 & 166.75 & 52.23 \\
Charges/interest & 8.20 & 9.65 & 5.53 & 2.10 & 0.29 \\
Total & 39.46 & 169.55 & 256.64 & 168.85 & 52.52
\end{tabular}

\section{Safeguard Assessments:}

An update of the December 2008 safeguards assessment of the National Bank of Georgia (NBG) was completed on January 21, 2010 in conjunction with an augmentation of access

\footnotetext{
${ }^{1}$ Following the Low Income Countries (LIC) reforms, effective January 7, 2010, the PRGF arrangements were renamed the Extended Credit Facility (ECF) Arrangements.
} 
under the SBA approved on August 6, 2009. The NBG continues to publish financial statements that comply with International Financial Reporting Standards, and are externally audited by an international firm in accordance with International Standards on Auditing. In response to the safeguards assessment, the authorities have agreed to a multi-year appointment of an external audit firm, beginning with financial year 2010 .

\section{Implementation of Multilateral Debt Relief Initiative (MDRI):}

Not Applicable.

\section{Implementation of HIPC Initiative:}

Not Applicable.

\section{Exchange Arrangements:}

(a) From 1993 to 2009, the National Bank of Georgia conducted foreign exchange market interventions through daily fixing sessions at the Tbilisi Interbank Currency Exchange (TICEX). A temporary de facto exchange rate peg to the U.S. dollar was introduced in the wake of the early August armed conflict. During the second week of November, the authorities allowed a 17 percent depreciation of the lari. In March 2009, the authorities introduced an auction-based system for the foreign exchange market. This mechanism is intended to allow more flexibility and give market forces a greater role in setting the price, with a view to reaching faster and smoother convergence toward equilibrium in the foreign exchange market and giving the market greater clarity about the authorities' policies. In endMay 2009, the authorities officially ended foreign exchange market interventions on TICEX.

(b) The government uses the official exchange rate for budget and tax accounting purposes as well as for all payments between the government and enterprises and other legal entities. The official rate may differ by more than two percent from freely determined market rates, which gives rise to a multiple currency practice. In practice, the official and market rates have never differed by more than 2 percent since the introduction of foreign exchange auctions in March 2009.

\section{Article IV Consultation:}

The 2011 Article IV consultation was concluded on March 23, 2011.

\section{FSAP Participation:}

Two FSAP missions visited Tbilisi during May 1-15, and July 24-August 7, 2001. An FSAP update mission visited Tbilisi during February 15-28, 2006.

\section{Technical Assistance:}

See Table 1 of this Annex. 


\section{Resident Representative:}

The sixth resident representative, Mr. Edward Gardner, took up his post on March 15, 2009.

\section{National Bank of Georgia Resident Advisors:}

Ms. Vance, MAE peripatetic banking supervision advisor to the NBG, commenced a series of visits to Tbilisi in September 1997. Mr. Nielsen, an MAE advisor, provided technical assistance to the NBG in May 1998. Mr. Viksnins was an MAE peripatetic advisor to the NBG president starting in October 1999. Mr. Fish was resident advisor on banking supervision from August 10, 1999 to January 31, 2002. Mr. Bernard Thompson provided peripatetic technical assistance in accounting and internal audit in March and August 2000. Mr. Wellwood Mason provided technical assistance on payment system issues on a peripatetic basis in 2002 and 2003. Mr. Howard C. Edmonds served from September 2004 to October 2007 as a resident advisor on banking supervision issues.

\section{Ministry of Finance Resident Advisors:}

Mr. Sharma was an FAD resident advisor and assisted the authorities in the development of a Treasury beginning in May 1997. Mr. Sainsbury, an FAD advisor, assisted the ministry of finance from June 1998 to November 1999. Mr. Chaturvedi was FAD resident advisor in 2001 and 2002 to assist the authorities in continuing the development of the Treasury and the Treasury Single Account, in revising the legislative framework, expenditure control systems, and budgeting issues. Between 2001 and 2003, Mr. Welling was an FAD peripatetic advisor to assist the State Customs Department in preparing and introducing measures for the custom reform and modernization program. In March 2005, Mr. Zohrab started advising the authorities on treasury-related reforms, and his term ended in November 2006. 
Table 1. Georgia: Fund Technical Assistance Missions, 2007-11

\begin{tabular}{llll}
\hline Subject & Type of Mission & Timing & Counterpart \\
\hline & \multicolumn{1}{c}{ Fiscal Affairs Department (FAD) } & \\
$\begin{array}{llll}\text { Tax Administration } \\
\text { Tax Administration }\end{array}$ & $\begin{array}{l}\text { Follow-up mission } \\
\text { Expert assistance } \\
\text { (Woodley) }\end{array}$ & $\begin{array}{l}\text { Jan. 17-30, 2007 } \\
\text { Jan/Feb and Apr/May }\end{array}$ & Ministry of Finance \\
Budget Classification and & $\begin{array}{l}\text { Expert assistance } \\
\text { (Swarap) }\end{array}$ & Jun. 11-22, 2007 & Ministry of Finance \\
$\begin{array}{l}\text { Accounting Reforms } \\
\text { Budget Classification and }\end{array}$ & $\begin{array}{l}\text { Expert assistance } \\
\text { Accounting Reforms }\end{array}$ & Jun. 11-22, 2007 & Ministry of Finance \\
$\begin{array}{l}\text { Public Financial } \\
\text { Management }\end{array}$ & Expert assistance & Apr. 6-19, 2010 & Ministry of Finance \\
Pension Reform & Expert assistance & Apr. 21-27, 2011 & Ministry of Finance
\end{tabular}

\section{Monetary and Capital Markets Department (MCM)}

Monetary

Operations/Monetary

Policy/Foreign Exchange

Consolidated Supervision

Lender of Last Resort

Framework

Stress Testing and

Foreign Exchange Market

Macroeconomic Modeling for Monetary Policy

Formulation

Macroeconomic Modeling for Monetary Policy

Formulation

Payment Systems

Oversight

Feb. 16-Mar. 2, 2007

National Bank of Georgia

Advisory

Dec. 4-14, 2007

National Bank of Georgia

Advisory

Dec. 11-17, 2008

National Bank of Georgia

Advisory

Apr. 21-30, 2009

Financial Supervisory

Agency; National Bank of Georgia

Advisory

June 2-10, 2010

National Bank of Georgia

Advisory

October 4-13, 2010

National Bank of Georgia

Advisory

May 3-12, 2011

National Bank of Georgia

\section{Statistics Department (STA)}

Balance of Payments

Follow-up assistance

Jul. 9-20, 2007

Monetary and Financial

Advisory

Mar. 18-31, 2009

National Bank of Georgia

National Bank of Georgia

Statistics

Evaluation of Technical Assistance

Follow-up assistance

Jul. 14-16, 2010

National Statistics Office, National Bank of Georgia, Ministry of Finance

External Sector Statistics 
Table 1. Georgia: Fund Technical Assistance Missions, 2007-11

\begin{tabular}{llll}
\hline Subject & Type of Mission & Timing & Counterpart \\
\hline & \multicolumn{1}{c}{ Legal Department (LEG) } \\
Payment Systems & Advisory & May 25-Jun. 5, 2010 & National Bank of Georgia \\
Payment Systems & Follow-up assistance & Nov. 8-14, 2010 & National Bank of Georgia \\
Legislative Drafting & Advisory & Feb. 28-Mar. 7, 2011 & National Bank of Georgia \\
\hline
\end{tabular}




\section{ANNEX II. GEORGIA: RELATIONS WITH THE WORLD BANK}

(As of May 10, 2011)

\begin{tabular}{|c|c|c|c|}
\hline Title & Product & $\begin{array}{l}\text { Tentative Timing of } \\
\text { Mission }\end{array}$ & $\begin{array}{l}\text { Expected Delivery of } \\
\text { Report }\end{array}$ \\
\hline Bank & $\begin{array}{l}\text { Operations } \\
\text { DPO program, including ongoing policy } \\
\text { dialogue on: } \\
\text { public investment management } \\
\text { program budgeting } \\
\text { social safety nets } \\
\text { tax and customs reforms } \\
\text { trade-related reforms } \\
\text { Public Sector Financial Management } \\
\text { Project Analytical Work } \\
\text { Programmatic PER } \\
\text { Trade Dialogue / Technical Assistance } \\
\text { Programmatic Poverty Assessment }\end{array}$ & $\begin{array}{l}\text { Ongoing } \\
\text { Quarterly } \\
\text { Ongoing } \\
\text { Periodic }\end{array}$ & $\begin{array}{l}\text { Ongoing } \\
\text { End-2010/Mid-2011 } \\
\text { Ongoing } \\
\text { Periodic }\end{array}$ \\
\hline Fund & $\begin{array}{l}\text { Missions and Technical Assistance: } \\
\text { Staff visit on } 2011 \text { budget and } \\
\text { macroeconomic framework update } \\
\text { SBA } 7^{\text {th }} \text { and } 8^{\text {th }} \text { Reviews } \\
\text { Art. IV Consultation } \\
\text { SBA } 9^{\text {th }} \text { Review } \\
\text { TA: payments system legal framework, } \\
\text { follow up } \\
\text { TA: payments system oversight } \\
\text { TA: macroeconomic model for monetary } \\
\text { policy analysis } \\
\text { TA: pensions } \\
\quad \text { Analytical work } \\
\text { DSGE model for monetary policy } \\
\text { analysis } \\
\text { Inflation process and forecast } \\
\text { Equilibrium exchange rate assessment } \\
\text { International reserve adequacy } \\
\text { assessment }\end{array}$ & $\begin{array}{l}\text { Sept.8-16, } 2010 \\
\text { Oct.27-Nov.10, } 2010 \\
\text { Feb.2-17, } 2011 \\
\text { Apr.12-28, } 2011 \\
\text { January } 2011 \\
\text { May 3-12, } 2011 \\
\text { Oct.4-14, } 2010 \\
\text { Jun.13-23, } 2011 \\
\text { Apr.21-27, } 2011\end{array}$ & $\begin{array}{l}\text { Sept.20, } 2010 \\
\text { Nov.15 (draft) } \\
\text { Feb.22 (draft) } \\
\text { May } 5 \text { (draft) } \\
\text { February } 2011 \\
\text { June } 2011 \\
\text { November } 2010 \\
\text { July } 2011 \\
\text { May } 2011\end{array}$ \\
\hline $\begin{array}{l}\text { Joint } \\
\text { Bank- } \\
\text { Fund }\end{array}$ & Joint Debt Sustainability Analysis & Ongoing collaboration & May 2011 \\
\hline
\end{tabular}




\title{
ANNEX III. GEORgIA: RELATIONS WITH THE EBRD
}

\author{
(As of April 30, 2011)
}

Since 1994, the EBRD has been active in supporting Georgia's transformation toward a market economy. The Bank's current country strategy for Georgia, approved in February 2010, outlines the following main strategic directions:

- Supporting stabilization and restructuring of the financial sector, strengthening risk and portfolio management practices and increasing the share of local currency operations;

- Helping rehabilitate and modernize the country's road, energy and municipal infrastructure to strengthen energy security and promote energy efficiency, help Georgia benefit from its potential as a transit country and improve efficiency and long-term financial sustainability of its municipal services;

- Provide financing to the enterprise sector with the view of supporting innovation, competition and export potential, in particular in manufacturing and agribusiness.

As of end of first quarter of 2011, the Bank had signed 131 investments in Georgia with the cumulative commitment totaling $€ 1$ billion. Its outstanding portfolio stood at $€ 692$ million. The majority of the portfolio is in the financial sector ( 40 percent), energy sector and infrastructure ( 25 percent and 21 percent, respectively). The ratio of private sector projects in the portfolio currently stands at 82 percent. The Bank gives preference to non-sovereign operations. Where sovereign guarantees are required, donor co-funding on a grant basis is sought.

In 2008-9, the Bank played an important role in stabilizing Georgia's financial system that had suffered from the August 2008 conflict and the global financial crisis. Along with the IFC, the EBRD provided Bank of Georgia and TBC Bank, the two largest systemic banks, with equity, quasi equity and loan facilities totaling $\$ 170$ million.

In 2010, the EBRD's operations focused on the infrastructure sector. The Bank signed several large projects, including Tbilisi Railway By-Pass ( $€ 112$ million), a sovereign guaranteed loan to finance the Black Sea Transmission Line (€80 million), the second stage of the rehabilitation of the Enghuri hydro power plant (€20 million) as well as a berth rehabilitation project at the Poti port ( $€ 8$ million) and Adjara solid waste landfill project ( $€ 3$ million).

The Bank also signed a number of smaller projects in the financial and industrial sectors. The Bank increased its equity investment in Bank Republic and provided SME credit lines to TBC Bank and Bank of Georgia ( $€ 5.6$ million and $€ 15$ million, respectively). It enhanced its Medium size Co-Financing Facility (MCFF) and signed four sub-projects in the manufacturing, agriculture and health sectors (totaling $€ 16$ million). MCFF has been working 
successfully for several years. The Bank also expanded the efforts in promoting its Energy Efficiency Initiative by providing $€ 3.7$ million facility for residential and industrial onlending to Bank of Georgia.

Georgia is part of the 'Early Transition Countries' (ETC) initiative. Launched in April 2004, the initiative aims to increase investments in the Bank's then seven poorest countries. The initiative builds on international efforts to address poverty in these countries. Through this initiative, the EBRD focuses its efforts on private sector business development and selected public sector interventions. It aims to stimulate market activity by using a streamlined approach to financing, focusing on smaller projects, mobilizing more investment, and encouraging ongoing economic reform. The Bank accepts higher risk in the projects it finances in the ETCs, while respecting the principles of sound banking. Since the launch of the ETC initiative, the Bank's annual business volume in Georgia has increased five-fold.

Going forward, the EBRD is actively engaged in promoting local currency lending and dedollarization of the Georgian banking system. Georgia is one of the first countries to undergo a joint EBRD-IMF-World Bank assessment of the economic policy, regulatory and legal frameworks for development of the local currency capital markets. On April 26 the Ministry of Finance and the National Bank of Georgia signed a Memorandum on Understanding with the EBRD in respect of macroeconomic and regulatory reforms to support dedollarization, paving the way for the expansion of EBRD lending in lari and technical assistance activities. 
Press Release No. 11/224

FOR IMMEDIATE RELEASE

June 9, 2011
International Monetary Fund

Washington, D.C. 20431 USA

\section{IMF Executive Board Completes Ninth and Final Review Under Stand-By Arrangement for Georgia}

On June 8, 2011, the Executive Board of the International Monetary Fund (IMF) completed the ninth and final review of Georgia's performance under the economic program supported by a Stand-By Arrangement.

Georgia's Stand-By Arrangement was approved by the Board on September 15, 2008 (see Press Release No. 08/208) for an amount equivalent to SDR 477.1 million (about US\$ 770 million $^{1}$ ). On August 6, 2009, the Executive Board approved an augmentation of access under the SBA to an amount equivalent to SDR 747.1 million (about US\$1.2 billion) and an extension of the SBA until June 14, 2011 (see Press Release No. 09/277). Between 2008 and July 2010, an amount equivalent to SDR 577.1 million (about US\$ 930 million) was disbursed under the SBA. Since then, the authorities have treated the arrangement as precautionary. The arrangement expires June 14, 2011.

In completing the review, the Board approved a waiver for the nonobservance of the performance criterion related to government expenditure.

Following the Executive Board's discussion on Georgia, Mr. Naoyuki Shinohara, Deputy Managing Director and Acting Chair, said:

"The Georgian authorities are to be commended for successfully implementing the SBAsupported program. The program objectives have been largely achieved, evidenced by the rebound in economic activity, improved market confidence, and the recovery of international reserves to their pre-crisis level. At the same time, the external adjustment process is not yet complete; public debt should be reduced further; and growth needs to be sustained to reduce unemployment. It will be important for the authorities' medium-term policy framework to address decisively these challenges.

\footnotetext{
${ }^{1}$ The amounts in U.S. dollars are calculated based on the SDR/US\$ exchange rate on June 7, 2011, with one U.S. dollar equal to SDR 0.61974.
} 
"The authorities are firmly committed to fiscal consolidation. It will be important to preserve sufficient flexibility to introduce revenue-enhancing measures, should the need arise.

Moreover, the planned increase in pensions should be structured and financed in a way that does not compromise the fiscal objectives.

"The authorities' current monetary policy stance is appropriate in the absence of secondround effects from the food and fuel price hikes. The authorities stand ready to adjust this stance promptly if signs of more persistent inflation emerge. The enhanced exchange rate flexibility achieved over the course of the program is commendable and should remain an anchor of the authorities' economic strategy.

"The banking sector has largely overcome the stress from the 2008 conflict and global financial crisis. Close monitoring for any signs of emerging new vulnerabilities in the sector will be important. The steps taken by the supervisory authority to strengthen its internal analytical capacity and responsiveness to risks are welcome." 


\section{Statement by Mr. Bakker, Executive Director for Georgia \\ and Mr. Lezhava, Advisor to Executive Director \\ June 8, 2011}

On behalf of my authorities, I would like to thank staff for the concise final report under the SBA that expires on June 14, 2011, and consistently constructive cooperation during the program period. The program allowed Georgia to provide an adequate response to the negative external shocks and to restore macroeconomic stability and confidence.

Macroeconomic indicators in general give reason for optimism; however, there still is a need for further adjustment.

To address the remaining challenges, the authorities are committed to their strategic principles of fiscal consolidation, flexible exchange rate, and inflation targeting regime. Fiscal consolidation will be achieved by keeping expenditure focused on targeted capital spending and allowing reasonable social outlays. The National Bank will remain committed to a flexible exchange rate and will aim at improving the transmission mechanism for inflation targeting. For monetary policy to be efficient, money market development, local currency credibility, lower dollarization, and improved modeling/forecasting capabilities are necessary. The National Bank is exploring opportunities for technical assistance in these areas with IFIs including the Fund.

\section{Economic Development}

Growth has returned to a positive path in almost all sectors, adding up to a 6.4 percent growth rate in 2010. Economic recovery was accompanied by some reduction in the unemployment rate to 16.3 percent in 2010 against 16.9 in 2009.

In 2010, the current account deficit declined to 9.6 percent of GDP, down from 22.6 percent in 2008. Part of this adjustment happened automatically, due to the reduced FDI related imports. As the staff report notices, further adjustment of the current account deficit is desirable. The main instrument for achieving greater competitiveness will be allowing adjustment of the exchange rate.

Exchange rate policy increasingly became more flexible during the program. In 2011 the National Bank intervened only occasionally in the foreign exchange market, mostly to purchase foreign exchange in line with the commitment to accumulate net international reserves.

Inflation is in the double digits and the authorities remain alert. Annual inflation has increased steadily from June 2010 onward, however, forecasts point to a substantial reduction in the second half of the year. As in many countries around the world, inflation is driven by 
high food and fuel prices, but core inflation remains subdued and no second-round effects have been observed so far.

The banking sector looks healthy with a gradually reducing trend of non-performing loans, increasing profits, comfortable liquidity, provisioning and capital levels, and a reasonable pace of credit growth.

The fiscal deficit was reduced from 9.2 in 2009 to 6.6 in 2010 and is expected to be lowered to 3.6 in 2011. A larger deficit reduction than previously envisaged seems to be possible, thanks to higher revenue collections.

\section{Future plans}

The authorities are facing the dilemma of needing to increase social spending and to reduce the fiscal deficit. In particular, the modalities of a pension increase are now under consideration. While the proposed increase of pension to the equivalent of USD 100 per month will likely impact overall budget outlays, the authorities remain committed to a steady and incremental path of budget deficit reduction.

Georgia has taken proactive steps to improve its debt sustainability. On April 7, 2011, Georgia successfully priced its 10-year benchmark Eurobond transaction, thereby achieving several important milestones for the country, in terms of the impressive pricing, very large oversubscription ratio, exceptionally low yield, and broadened investor base. Georgia was able to increase the maturity from 5 to 10 years, while lowering the yield from 7.5 to 7.125. As a result, the authorities successfully addressed the challenge of a public debt service spike in 2013. The Ministry of Finance remains active also in the local market, by gradually increasing the maturity of government papers. It is committed to a conservative public debt management vision, to preclude shrinkage of the fiscal space and secure sustainable debt dynamics. This is of the essence given the timeline for redemptions under the current StandBy Arrangement with the IMF, which come due in 2011-2015.

In order to maintain market confidence, secure access to Fund resources in the event of external financing needs, and be able to benefit from continuous advice, the authorities are looking forward to further engagement with the Fund. Therefore, Georgia will request a successor program once the specific details of the medium-term policy framework have been formulated.

The Georgian authorities are thankful to the IMF board members for their comments and suggestions expressed during the program discussions. These inputs received serious attention and were reflected in policy decisions taken during the program. Commitment to the above mentioned fiscal and monetary strategic principles will continue to guide policy decisions going forward. 